\title{
Mechanistic roles of tyrosine phosphorylation in reversible amyloids, autoinhibition, and endosomal membrane association of ALIX
}

Received for publication, July 28, 2021, and in revised form, October 5, 2021 Published, Papers in Press, October 22, 2021,

https://doi.org/10.1016/j.jbc.2021.101328

\author{
Ruben D. Elias ${ }^{\circ}$, Bhargavi Ramaraju $\odot$, and Lalit Deshmukh*(it) \\ From the Department of Chemistry and Biochemistry, University of California San Diego, La Jolla, California, USA
}

Edited by Wolfgang Peti

Human apoptosis-linked gene-2 interacting protein $X$ (ALIX), a versatile adapter protein, regulates essential cellular processes by shuttling between late endosomal membranes and the cytosol, determined by its interactions with Src kinase. Here, we investigate the molecular basis of these transitions and the effects of tyrosine phosphorylation on the interplay between structure, assembly, and intramolecular and intermolecular interactions of ALIX. As evidenced by transmission electron microscopy, fluorescence and circular dichroism spectroscopy, the proline-rich domain of ALIX, which encodes binding epitopes of multiple cellular partners, formed rope-like $\beta$-sheet-rich reversible amyloid fibrils that dissolved upon Srcmediated phosphorylation and were restored on proteintyrosine phosphatase 1B-mediated dephosphorylation of its conserved tyrosine residues. Analyses of the Bro1 domain of ALIX by solution NMR spectroscopy elucidated the conformational changes originating from its phosphorylation by Src and established that Bro1 binds to hyperphosphorylated proline-rich domain and to analogs of late endosomal membranes via its highly basic surface. These results uncover the autoinhibition mechanism that relocates ALIX to the cytosol and the diverse roles played by tyrosine phosphorylation in cellular and membrane functions of ALIX.

Human apoptosis-linked gene-2 interacting protein $\mathrm{X}$ (ALIX) is a conserved adapter protein involved in essential cellular processes, including endosomal protein sorting, apoptosis, exosome biogenesis, and many others (1-4). ALIX also regulates budding of diverse enveloped viruses, such as HIV $(5,6)$ and Ebola (7). It comprises an amino (N)-terminal Bro1 domain (residues 1-359), a central coiled-coil domain termed V (residues 360-702), and a carboxy (C)-terminal proline-rich domain (PRD; residues 703-868) (Fig. 1A). ALIX, also known as programmed cell death 6 interacting protein (PDCD6IP), undergoes tyrosine phosphorylation at multiple sites, which regulates its cellular functions; it comprises 28 conserved tyrosine residues (8), among which 11 are localized in the C-terminal portion of PRD, residues 803 to 846 . ALIX interacts with and is phosphorylated by Src kinase at late

\footnotetext{
*For correspondence: Lalit Deshmukh, Ideshmukh@ucsd.edu.
}

endosomal membranes (8, 9); ALIX-endosomal membrane interactions are governed by its Brol domain, which binds to negatively charged $2,2^{\prime}$ lysobisphosphatidic acid (2,2' LBPA; referred to as LBPA), a phospholipid found in late endosomes (10-12). ALIX-Src interactions result in phosphorylation of the Bro1 domain and hyperphosphorylation of the tyrosinerich portion of $\operatorname{PRD}(8,9)$. These interactions promote exosome secretion and cause ALIX to relocate from a membrane-bound active conformation to a cytosolic inactive state. Interdomain interactions between PRD and the upstream globular domains, Bro1 and V, are suggested to cause ALIX autoinhibition in the cytosol $(13,14)$. Structural details of these interactions are unclear because of the unavailability of recombinant full-length ALIX, owing to difficulties in expressing ALIX-PRD in bacteria and its disordered nature. Intact ALIX was, however, successfully produced using a baculovirus-insect cell expression system (14). ALIX-PRD encodes binding epitopes of numerous cellular proteins (15), and its hyperphosphorylation by Src affects its cellular interactions (8). We recently discovered that the $\mathrm{N}$-terminal portion of ALIX-PRD (residues 703-800) is disordered, whereas its C-terminal tyrosine-rich portion (residues 800-868) forms amyloid fibrils that disassemble on Srcmediated hyperphosphorylation (16). However, the mechanistic effects of tyrosine phosphorylation on cellular redistribution and functions of ALIX are unclear.

Using recombinant ALIX domains and a range of biophysical methods, including NMR, fluorescence, and circular dichroism (CD) spectroscopy, mass spectrometry (MS), transmission electron microscopy (TEM), and dynamic light scattering (DLS), we elucidate how tyrosine phosphorylation affects the interplay between ALIX conformation, assembly, and function (poor bacterial expression and the overall size, $\sim 96 \mathrm{kDa}$, do not allow for a similar study in the context of full-length ALIX). Codon optimization coupled with a single-point mutation permitted expression of full-length ALIX-PRD in Escherichia coli. We show that PRD forms reversible $\beta$-sheet-rich fibrils modulated by posttranslational modifications (PTMs), Src-mediated phosphorylation, and human protein-tyrosine phosphatase $1 \mathrm{~B}$ (PTP1B)-mediated dephosphorylation of its conserved tyrosine residues. We establish that Bro1 binds to hyperphosphorylated PRD and to analogs of late endosomal membranes. The close correlation between the regions of Brol that associate with 


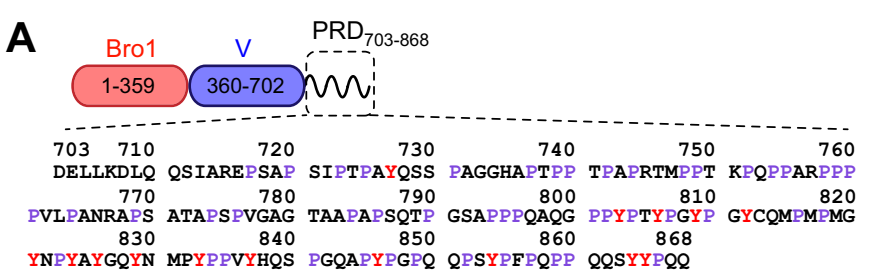

B

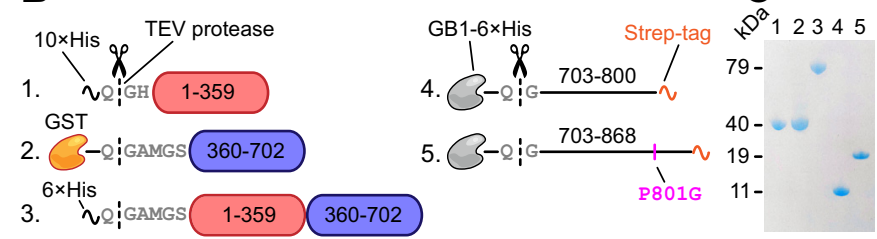

Figure 1. ALIX domain organization and summary of recombinant ALIX constructs used in the current work. $A$, schematic of ALIX organization. Primary sequence of the PRD is shown. $B$, recombinant ALIX constructs used in the current work. Constructs 1 to 3 were obtained from Addgene; accession no. 80641 (47), 17639 (48), and 42577 (49), respectively. Construct 4 is described in our previous work (16); Addgene accession no. 141344. The positions of purification tags are marked. TEV protease cutting sites are shown. The point mutation, P801G, of PRD analysis of TEV-cleaved ALIX constructs; the order of the constructs is the same as the one depicted in B. ALIX, apoptosis-linked gene- 2 interacting protein X; PRD, proline-rich domain; TEV, tobacco etch virus.

hyperphosphorylated PRD and with anionic phospholipids elucidates the underlying mechanism of PRD-mediated autoinhibition of ALIX-membrane interactions and how tyrosine phosphorylation causes redistribution of ALIX away from late endosomal membranes into the cytosol.

\section{Results}

\section{Recombinant constructs}

The current work made use of five recombinant ALIX constructs (Fig. 1, $B$ and $C$ ). These include constructs representing the individual Bro1 and V domains (residues 1-359 and 360-702, respectively), a construct comprising both the Bro1 and $\mathrm{V}$ domains (residues 1-702; referred to as Bro1-V), and two constructs representing the $\mathrm{N}$-terminal portion of PRD, PRD Strep $\mathrm{PRD}_{703-868}^{\text {Strep }}$ (residues 703-868); "Strep" denotes a noncleavable C-terminal strep-affinity tag (17) that facilitated purification of intact PRD constructs from their truncated fragments using affinity chromatography. The latter are generated during heterologous expression of ALIX-PRD in $E$. coli because of translational arrest induced by its polyproline motifs. $\mathrm{PRD}_{703-868}^{\text {Strep }}$ carries a P801G point mutation to resolve issues with protein expression in E. coli. We previously established that the ${ }^{800}$ GPPYP $^{804}$ segment of ALIX-PRD is responsible for ribosomal stalling in $E$. coli, resulting in essentially no expression of wildtype PRD beyond residue 801 (16). Note that the effects of the P801G mutation on the cellular functions of ALIX are currently not known. For paramagnetic NMR, three cysteine variants of $\mathrm{PRD}_{703-868}^{\text {Strep }}$ were engineered (see below). We also made use of full-length Src kinase and the catalytic domain of PTP1B; see Figure S1 for liquid chromatography (LC)-electrospray ionization (ESI)-time-of-flight (TOF)MS analyses of constructs used in the current study.

\section{Reversible amyloid fibrils of $\mathrm{PRD}_{703-868}^{\text {Strep }}$}

Using dye-binding assays with amyloid-specific probes, thioflavin $\mathrm{T}$ (ThT) and congo red (CR), and TEM, we previously established that the C-terminal tyrosine-rich fragment of ALIX-PRD (PRD ${ }_{800-868}$ ) forms rope-like fibrils that dissolve into monomeric units on Src-mediated hyperphosphorylation (16). Because of the poor solubility of $\mathrm{PRD}_{800-868}(<30 \mu \mathrm{M} ; \mathrm{pH}$ $4.5-7)$, the data reported in our prior study were collected on a fusion protein with an $\mathrm{N}$-terminal solubility enhancement tag comprising the B1 domain of protein $\mathrm{G}$ (GB1) (18). In contrast to $\mathrm{PRD}_{800-868}, \mathrm{PRD}_{703-868}^{\text {Strep }}$ was more soluble $(\sim 300 \mu \mathrm{M} ; \mathrm{pH}$ 4.5-8), which allowed us to characterize its aggregation properties and its interactions with Src in the absence of a GB1 tag. LC-ESI-TOFMS analyses of Src-mediated phosphorylation revealed the formation of hyperphosphorylated $\mathrm{PRD}_{703-868}^{\text {Strep }}$ with phosphorylation of six to ten (out of 15) tyrosine residues (Fig. $2 A$ and Table S1). ThT emission assays confirmed the presence of $\beta$-sheet-rich conformations for $\mathrm{PRD}_{703-868}^{\text {Strep }}$ aggregates (Fig. $2 B$ ). Spectral-shift assays carried out using CR demonstrated clear shifts toward $540 \mathrm{~nm}$, further validating their amyloidogenic nature (Fig. $2 C$ ). For these assays, the soluble N-terminal fragment, $\mathrm{PRD}_{703-800}^{\text {Strep }}$ (16) and hyperphosphorylated $\mathrm{PRD}_{703-868}^{\text {Strep }}$ served as negative controls. NMR analysis revealed a near-perfect superimposition of ${ }^{1} \mathrm{H}-{ }^{15} \mathrm{~N}$ transverse relaxation optimized spectroscopy (TROSY)-heteronuclear single quantum coherence (HSQC) spectra of these two proteins, establishing Src-mediated dissolution of $\mathrm{PRD}_{703-868}^{\text {Strep }}$ aggregates into NMR-amenable soluble monomers and the complete lack of interactions between the $\mathrm{N}$-terminal and C-terminal portions of PRD (Fig. S2). Backbone resonance assignments of hyperphosphorylated $\mathrm{PRD}_{703-868}^{\text {Strep }}$ were not feasible owing to the complexity and heterogeneity of its phosphorylation pattern. However, a narrow chemical shift dispersion ( $7.7-8.7 \mathrm{ppm})$ suggested that the hyperphosphorylated $\mathrm{PRD}_{703-868}^{\text {Strep }}$ is disordered in solution. TEM analyses revealed the presence of rope-like unbranched $\mathrm{PRD}_{703-868}^{\text {Strep }}$ filaments, characteristic of amyloid fibrils (Fig. 2D). CD spectroscopic data confirmed the presence of $\beta$-sheet conformations in these fibrils (Fig. 2E), corroborating the presence of amyloid structure. The corresponding CD spectrum of hyperphosphorylated $\mathrm{PRD}_{703-868}^{\text {Strep }}$ validated its random-coil conformation.

To identify the fibril core resistant to protease treatment, a limited digestion of $\mathrm{PRD}_{703-868}^{\text {Strep }}$ fibrils was carried out using proteinase $\mathrm{K}$ (PK) (Fig. 2, F and G). Based on band intensities of the reaction mixture comprising intact protein and the proteolyzed products monitored using SDS-PAGE (Fig. 2F), we conclude that $\mathrm{PRD}_{703-868}^{\text {Strep }}$ fibrils are resistant to PK digestion as the band for $\mathrm{PRD}_{703-868}^{\text {Strep }}$ persisted after $\sim 60 \mathrm{~min}$ of incubation with PK, albeit at a lower intensity than the corresponding band at time 0 . In contrast, the soluble $\mathrm{PRD}_{703-800}^{\mathrm{Strep}}$ was completely hydrolyzed by PK within $\sim 5$ min of the reaction. LC-tandem MS (LC-MS/MS) analysis of PK digestion of $\mathrm{PRD}_{703-868}^{\text {Strep }}$ fibrils revealed that the two most abundant fragments were localized in the $\mathrm{C}$-terminal region, residues 800 to 813 and 840 to 863 (Figs. $2 G$ and S3), indicating that these motifs likely participate in the formation of the fibril core. A 
A

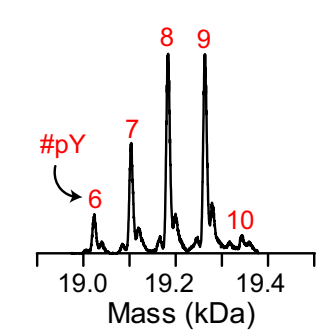

$\mathbf{F}$

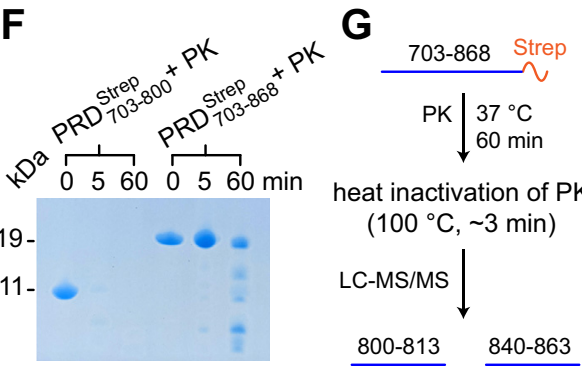

B

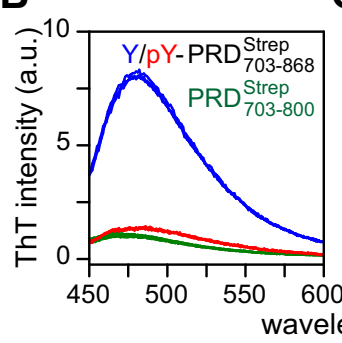

$\mathrm{H}$
C

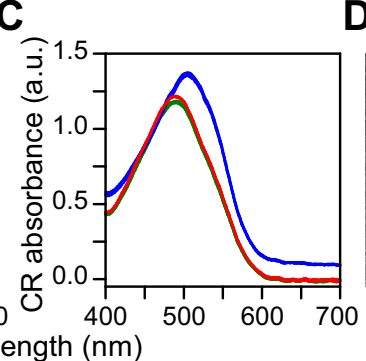

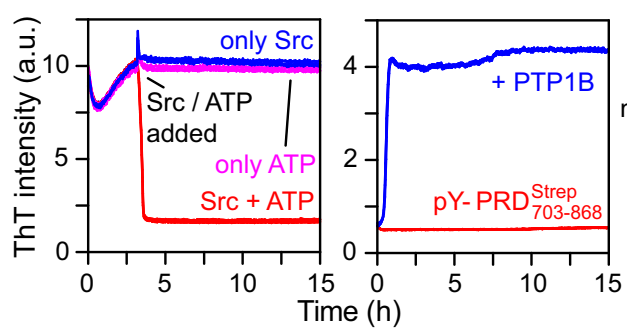

E

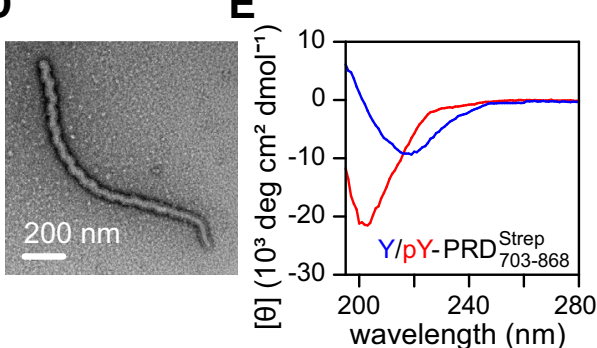

J

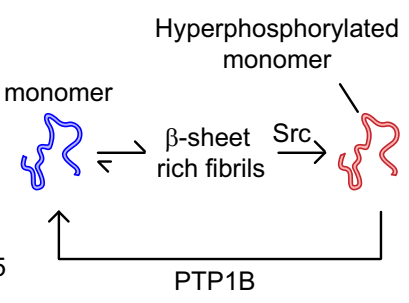

Figure 2. Reversible amyloids of PRD $_{\mathbf{7 0 3}-\mathbf{8 6 8}}^{\text {Stre }}$. A, LC-ESI-TOFMS analysis of Src-mediated phosphorylation of PRD ${ }_{703-868}^{\text {Strep }}$ the number of phosphorylated tyrosine residues is marked in red. Emission spectra of ThT, a.u., arbitrary units $(B)$ and absorbance spectra of $C R$, a.u., absorbance units $(C)$ of PRD ${ }_{703-868}^{\text {Strep }}$ aggregates (blue); $\mathrm{n}=3$. $\mathrm{PRD}_{703-800}^{\text {Strep }}$ (green) and hyperphosphorylated $\mathrm{PRD}_{703-868}^{\text {Strep }}$ (red) were used as controls. $D$, negatively stained EM images of PRD fibrils. $E, C D$ spectra of PRD Prtre $_{7038}$ fibrils (blue) and its hyperphosphorylated form (red). F, SDS-PAGE analysis of limited PK digestion of PRD Strep ${ }_{703}$ fibrils and monomeric PRD $\mathrm{PR}_{703-800}^{\text {Strep }}$ G, scheme of PK digestion and the results of LC-MS/MS analysis. $H$ and l, the impact of tyrosine phosphorylation and dephosphorylation on aggregation kinetics of $\mathrm{PRD}_{703-868}^{\text {Strep }}$ assessed using ThT assays $\left(30^{\circ} \mathrm{C}\right) . \mathrm{H}, 100 \mu \mathrm{M} \mathrm{PRD}_{703-868}^{\text {Strep }}$ was incubated without $\mathrm{Src}$ for $\sim 3 \mathrm{~h}(\mathrm{n}=9)$. $10 \mu \mathrm{M}$ Src $+1 \mathrm{mM}$ ATP were added to three samples (red), whereas the remaining received either $10 \mu \mathrm{M}$ Src (blue) or $1 \mathrm{mM}$ ATP (pink). l, $100 \mu \mathrm{M}$ hyperphosphorylated PRD $\mathrm{Pr}_{703-868}^{\text {step }}$ samples $(\mathrm{n}=3)$ were incubated in the absence $(r e d)$ and the presence (blue) of $50 \mathrm{nM}$ PTP1B. J, scheme depicting potential mode of formation and dissolution of $\mathrm{PRD}_{703-868}^{\text {Strep }}$ fibrils. $\mathrm{CR}$, congo red; ESI, electrospray ionization; PK, proteinase K; PRD, proline-rich domain; PTP1B, proteintyrosine phosphatase $1 \mathrm{~B}$; ThT, thioflavin T.

limited PK digestion (incubation time of $\sim 5 \mathrm{~min}$ ) was also performed on hyperphosphorylated $\mathrm{PRD}_{703-868}^{\text {Strep }}$, and the corresponding cleavage products were identified using LC-MS/ MS, which permitted identification of phosphorylated tyrosine residues, namely $803,806,809,812,829,833,837,846$, and 854 (Fig. S4). Nothing definitive can be said about the phosphorylation status of the remaining tyrosine residues because of sequencing coverage gaps arising from problems associated with proteolytic digestion of $\mathrm{PRD}_{703-868}^{\text {Strep }}$. Note that the tyrosine-rich portion of $\mathrm{PRD}_{703-868}^{\text {Strep }}$, residues 800 to 868 , carries no native lysine and arginine residues ( $c f$., Fig. $1 A$ ) and, thus, cannot be probed by traditional trypsin-based proteomics (19). PK digestion of hyperphosphorylated PRD Ptrep $_{708}^{\text {Ste was not }}$ analyzed by SDS-PAGE because of smearing of protein bands owing to phosphorylation (20).

To assess the impact of tyrosine phosphorylation on $\mathrm{PRD}_{703-868}^{\text {Strep }}$ polymerization, aggregation kinetics using ThT fluorescence were monitored. $\mathrm{PRD}_{703-868}^{\text {Strep }}$ exhibited sigmoidal aggregation profiles, a hallmark of fibril formation (Fig. $2 H$ ). To establish Src-mediated dissolution of PRD $_{703-868}^{\text {Strep }}$ fibrils, Src and ATP were added to PRD $_{703-868}^{\text {Strep }}$ samples upon reaching a stationary phase (at $\sim 3 \mathrm{~h}$; Fig. $2 H$ ), which resulted in a significant loss of ThT signal with a corresponding half-life $\left(t_{1 / 2}\right)$ of $\sim 0.2 \mathrm{~h}$. The remaining PRD $_{703-868}^{\text {Strep }}$ samples were mixed with either Src or ATP and displayed no drop in ThT signals, indicating that Src or ATP do not affect $\mathrm{PRD}_{703-868}^{\text {Strep }}$ fibrils. Remarkably, judging by the rapid appearance of characteristic sigmoidal ThT profiles $\left(t_{1 / 2} \sim 0.5 \mathrm{~h}\right.$; Fig. $\left.2 I\right)$, dephosphorylation of hyperphosphorylated $\mathrm{PRD}_{703-868}^{\text {Strep }}$ by PTP1B resulted in restoration of fibrils, whereas the hyperphosphorylated PRD $_{703-868}^{\text {Strep }}$ without PTP1B showed no sigmoidal ThT profile (activity of recombinant PTP1B was measured using the malachite green assay; Fig. S5. For Src, the activity was measured using the ADP-Glo assay, described in our previous work (16)). Although the exact identity of the phosphatase that works in tandem with ALIX is not known, PTP1B was suggested to regulate the endosomal sorting machinery (21). These observations establish that $\mathrm{PRD}_{703-868}^{\text {Strep }}$ forms rope-like $\beta$-sheet-rich fibrils that dissolve into soluble monomers on hyperphosphorylation by Src and that the removal of phosphoryl groups via PTP1B culminates in the restoration of fibrils, making $\mathrm{PRD}_{703-868}^{\text {Strep }}$ a completely reversible amyloid controlled by PTMs (Fig. 2J). In addition to PRD, Src is suggested to phosphorylate the Bro1 domain of ALIX in vivo (8). To characterize the impact of phosphorylation on Brol, we performed detailed NMR analyses of nonphosphorylated and phosphorylated Bro1, described below.

\section{NMR analysis of nonphosphorylated and phosphorylated Bro1}

ALIX-Src interactions are suggested to proceed in a stepwise fashion in vivo, where Src first binds and phosphorylates the highly conserved region, ${ }^{312} \mathrm{KKDNDFIY}^{319}$, of the Bro1 domain, followed by hyperphosphorylation of the tyrosine-rich portion of PRD, ultimately resulting in the relocation of ALIX from late endosomal membranes to the cytosol (8). Srcmediated phosphorylation of Bro1 was confirmed using 


\section{Structure-function studies of tyrosine phosphorylation of ALIX}

Western blotting (Fig. S6A). LC-ESI-TOFMS and LC-MS/ MS analyses established the formation of phosphorylated Bro1 and confirmed the phosphorylation of Y319 (Fig. S6, B and C, respectively). To explore the impact of tyrosine phosphorylation on Bro1 at atomic resolution, we conducted a detailed NMR investigation (Fig. 3). Although the overall size of Bro1 is relatively large for solution NMR studies $(\sim 40 \mathrm{kDa})$, excellent spectral quality was obtained by perdeuteration coupled with TROSY (Fig. $3 A$ ), which allowed us to carry out nearly complete backbone resonance assignments of Bro1. Based on an excellent agreement between the secondary structure derived from the assigned backbone chemical shifts $\left({ }^{13} \mathrm{C} \alpha,{ }^{13} \mathrm{C} \beta,{ }^{13} \mathrm{C}^{\prime}\right.$, ${ }^{15} \mathrm{~N}$, and ${ }^{1} \mathrm{H}_{\mathrm{N}}$ ) using TALOS-N (22) with that obtained from the three-dimensional crystal structure of Bro1 (Protein Data Bank entry: 5WA1 (23); Fig. S7), we conclude that recombinant Bro1 is well folded in solution. To probe structural and conformational changes that take place in Bro1 upon Srcmediated phosphorylation of $\mathrm{Y} 319,{ }^{1} \mathrm{H}_{\mathrm{N}} /{ }^{15} \mathrm{~N}$ chemical shift perturbation mapping was utilized. NMR analyses of phosphorylated Brol revealed large ${ }^{1} \mathrm{H}_{\mathrm{N}} /{ }^{15} \mathrm{~N}$ chemical shift perturbations that arise from the introduction of a negatively charged phosphoryl group (Fig. 3B). Mapping of these perturbations onto the crystal structure of Bro1 allowed identification of regions affected by tyrosine phosphorylation (Fig. 3C). All perturbations were localized in the regions surrounding Y319, which further confirmed specific phosphorylation of Bro1 by Src. The heteronuclear ${ }^{15} \mathrm{~N}-\left\{{ }^{1} \mathrm{H}\right\}$ NOE data (Fig. 4) of nonphosphorylated and phosphorylated Bro1 indicated that the introduction of phosphoryl group did not affect the local internal mobility of Bro1. Collectively, the aforementioned data established that Src-mediated phosphorylation of Bro1 at Y319 induces local changes.

\section{Lack of interactions between PRD ${ }_{703-800}^{\text {Strep }}$ and globular ALIX domains}

A prior study suggested that the $\mathrm{N}$-terminal portion of PRD, specifically the ${ }^{717}$ PSAP $^{720}$ motif that interacts with the ubiquitin E2 variant (UEV) domain of tumor-susceptibility gene 101, directly associates with the Src-binding region of Bro1 (13). PRD was also suggested to prevent the binding between the $\mathrm{V}$ domain and the $\mathrm{YPX}_{\mathrm{n}} \mathrm{L}$ consensus motifs $(\mathrm{X}=$ any residue and $n=1-3$ ) found in viral proteins, although molecular details of this inhibition were not determined (14). To investigate interdomain interactions of ALIX, we carried out ${ }^{1} \mathrm{H}_{\mathrm{N}} /{ }^{15} \mathrm{~N}$ chemical shift perturbation mapping using $\mathrm{PRD}_{703-800}^{\text {Strep }}$ and nonphosphorylated and phosphorylated Bro1 (Fig. 5). Negligible ${ }^{1} \mathrm{H}_{\mathrm{N}} /{ }^{15} \mathrm{~N}$ chemical shift perturbations were observed for both nonphosphorylated and phosphorylated NMR-visible Bro1, one at a time, upon the addition of threemolar equivalents of $\mathrm{PRD}_{703-800}^{\text {Strep }}$ (Fig. 5, $A$ and $B$, respectively), indicating that Brol did not bind to $\mathrm{PRD}_{703-800}^{\mathrm{Strep}}$. The corresponding titration experiments carried out using NMR-visible PRD $_{703-800}^{\text {Strep }}$ and unlabeled nonphosphorylated and phosphorylated Bro1 yielded negligible chemical shift changes (Fig. $5 C$ ), confirming the lack of Bro1-PRD ${ }_{703-800}^{\text {Strep }}$ interactions. To determine the involvement of the $\mathrm{V}$ domain, titration experiments were carried out using NMR-visible $\mathrm{PRD}_{703-800}^{\text {Strep }}$, in the absence and presence of three-molar equivalents of $\mathrm{V}$ and Bro1-V constructs (one at a time). Minimal chemical shift
A

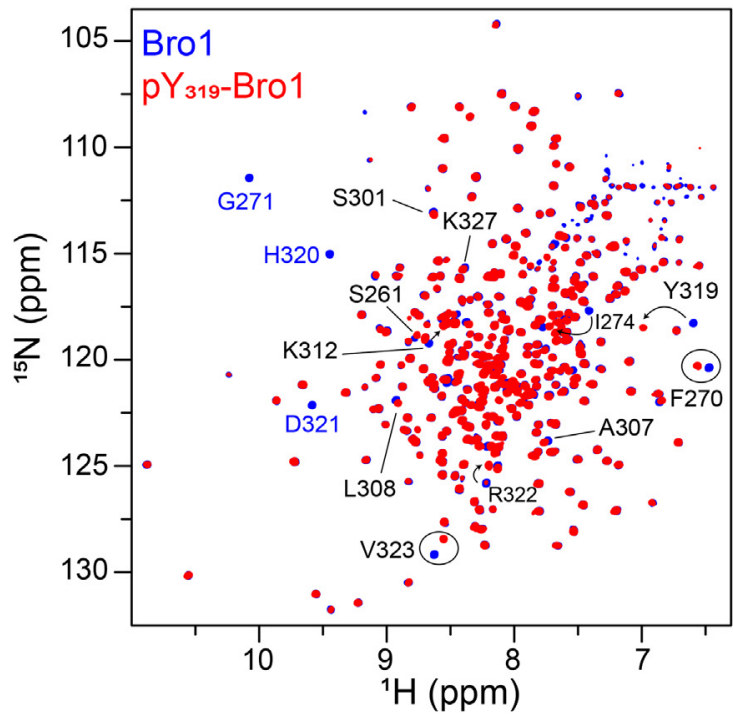

B

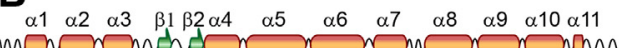

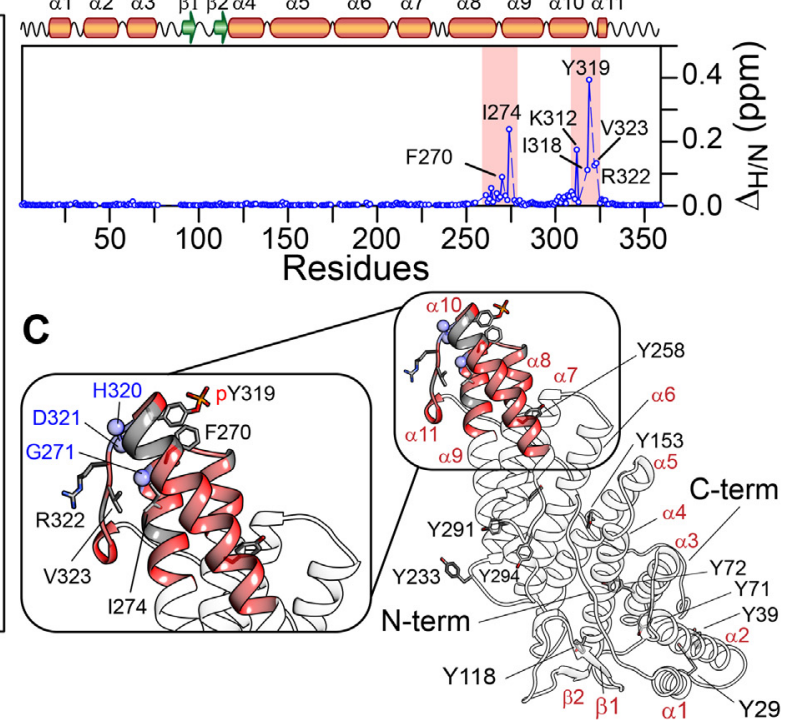

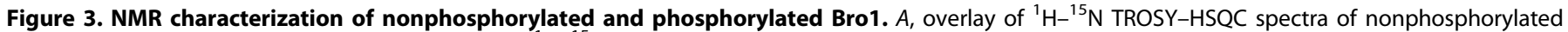

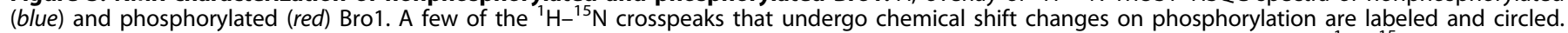

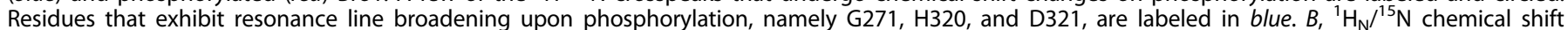

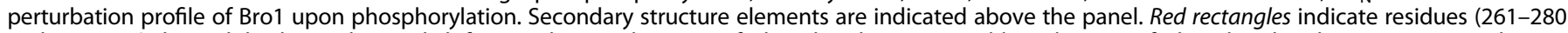

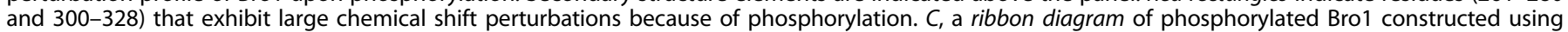

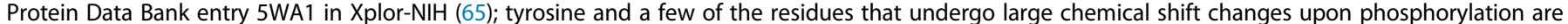

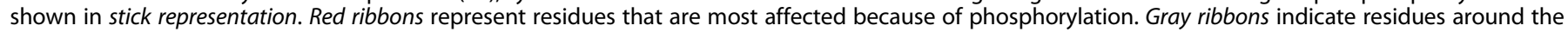

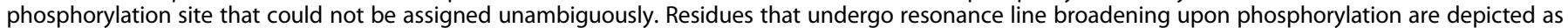
blue spheres. HSQC, heteronuclear single quantum coherence; NIH, National Institutes of Health; TROSY, transverse relaxation optimized spectroscopy. 


\section{Structure-function studies of tyrosine phosphorylation of ALIX}

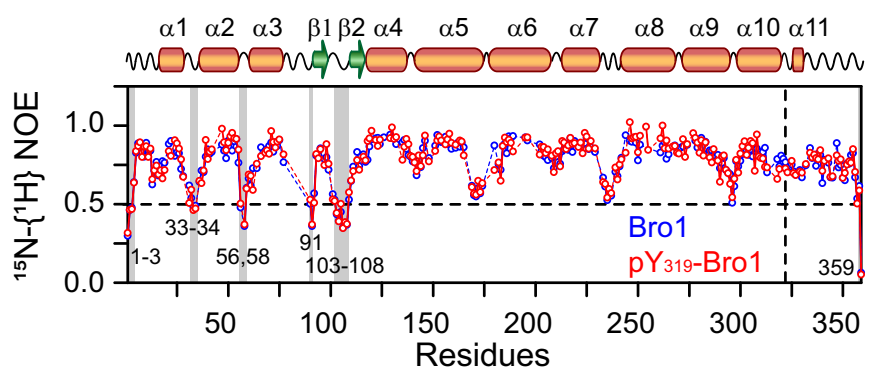

Figure 4. ${ }^{15} \mathrm{~N}-\left\{{ }^{1} \mathrm{H}\right\}$ heteronuclear NOE data for nonphosphorylated and phosphorylated Bro1. Regions with high local mobility on the nanosecond time scale, ${ }^{15} \mathrm{~N}-\left\{{ }^{1} \mathrm{H}\right\} \mathrm{NOE}<0.5$, are labeled and marked with semitransparent gray rectangles. The location of $\mathrm{Y} 319$ is indicated by a vertical dashed line. All data were acquired with $500 \mu \mathrm{M}{ }^{2} \mathrm{H} /{ }^{15} \mathrm{~N}$ labeled proteins at a spectrometer ${ }^{1} \mathrm{H}$ frequency of $800 \mathrm{MHz}$ at $30^{\circ} \mathrm{C}$.

changes indicated that $\mathrm{V}$ and Bro1-V constructs did not bind to PRD $703-800$ (Fig. 5C). Experiments using NMR-visible V and Bro1-V constructs were not viable since both produced poorquality NMR spectra, likely because of the open-close transitions of the V domain in solution (14). Collectively, these observations indicated that Bro1 and $\mathrm{V}$ domains did not interact with $\mathrm{PRD}_{703-800}^{\mathrm{Strep}}$. These findings are consistent with the fact that unlike proline-recognition domains such as UEV $(24,25)$, Bro1 domains have not been known to bind to proline-rich motifs, and that ALIX-PRD carries no $\mathrm{YPX}_{\mathrm{n}} \mathrm{L}$ consensus motif recognized by the $\mathrm{V}$ domain. Finally, nothing definitive can be said about the interactions of intact $\mathrm{PRD}_{703-868}^{\text {Strep }}$ with Bro1 and V domains in solution because of its propensity to form fibrils. We, however, uncovered novel interactions between Bro1 and hyperphosphorylated $\mathrm{PRD}_{703-868}^{\text {Strep }}$, which are described below.

\section{Interactions between Bro1 and hyperphosphorylated PRD $_{703-868}^{\text {Strep }}$}

Src-mediated hyperphosphorylation of PRD relocates ALIX from late endosomal membranes to the cytosol (8), indicating a likely competition between hyperphosphorylated PRD and membranes for association with the phospholipids-binding region(s) of Bro1. To test this hypothesis and identify contacts between Bro1 and hyperphosphorylated $\mathrm{PRD}_{703-868}^{\text {Strep }}$, we made use of intermolecular paramagnetic relaxation enhancement (PRE) (26). The magnitude of the PRE effect is very large owing to the large magnetic moment of the unpaired electron of the paramagnetic label and is proportional to the $<\mathrm{r}^{-6}>$ paramagnetic label-proton distance, which enables the detection of sparsely populated (as low as $0.5-1 \%$ in favorable cases) encounter complexes between binding partners (27). The paramagnetic nitroxide spin label (1-oxyl-2,2,5,5tetramethyl- $\Delta 3$-pyrroline-3-methyl) methanethiosulfonate (MTSL) was introduced at four separate sites on hyperphosphorylated $\mathrm{PRD}_{703-868}^{\text {Strep }}$ via disulfide linkages (Fig. S8A; also see Fig. S8B and Table S1 for LC-ESI-TOFMS analysis of MTSL conjugated products). These four sites were the native cysteine residue of $\mathrm{PRD}_{703-868}^{\text {Strep }}, \mathrm{C} 813$, and three engineered sites, S712C, A756C, and S863C (Fig. 6A; in all cysteine variants, C813 was mutated to a serine). Site C813 lies adjacent to

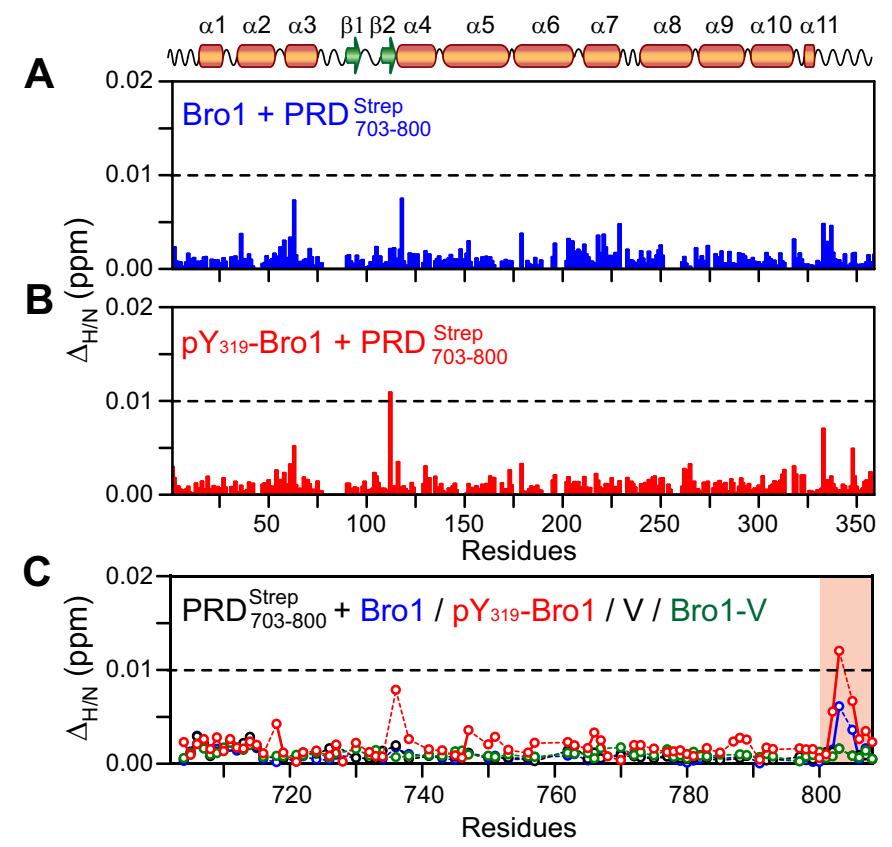

Figure 5. Lack of association between PRD $_{703-800}^{\text {Strep }}$ and globular ALIX domains. ${ }^{1} \mathrm{H}_{\mathrm{N}} /{ }^{15} \mathrm{~N}$ chemical shift perturbation profiles of $(A)$ nonphosphorylated and $(B)$ phosphorylated ${ }^{2} \mathrm{H} /{ }^{15} \mathrm{~N}$-labeled Bro1 on addition of three-molar equivalent of nonlabeled $\mathrm{PRD}_{703-800}^{\text {Strep }} . C^{1}{ }^{1} \mathrm{H}_{\mathrm{N}} /{ }^{15} \mathrm{~N}$ chemical shift perturbation profiles of ${ }^{15} \mathrm{~N}$-labeled $\mathrm{PRD}_{703-800}^{\text {Strep }}$ on addition of nonlabeled Bro1 (blue), phosphorylated Bro1 (red), V (black), and Bro-V (dark green); one at a time, $\mathrm{PRD}_{703-800}^{\text {Strep }}$ to globular ALIX domains molar ratio $=1: 3$. Region highlighted in semitransparent orange rectangle indicates the location of the C-terminal strep purification tag (residues 801-808). ALIX, apoptosis-linked gene-2 interacting protein $\mathrm{X}$; PRD, proline-rich domain.

a cluster of tyrosine residues that are phosphorylated by Src (cf., Fig. S4). Sites S712C and A756C are located near the motif that binds to tumor-susceptibility gene 101-UEV (residues $717-720 ;(16)$ ) and in the highly basic region of PRD (residues $756-767$; theoretical isoelectric point $\sim 12$ ), respectively. Site S863C is located at the extreme C terminus of PRD and is adjacent to ${ }^{864} \mathrm{YY}^{865}$ (cf., Fig. $1 A$ ); we were unable to detect the phosphorylation status of these two tyrosine residues because of sequencing coverage gaps.

Intermolecular PRE $\left({ }^{1} \mathrm{H}_{\mathrm{N}}-\Gamma_{2}\right)$ profiles observed for NMRvisible Bro1 in the presence of paramagnetically labeled hyperphosphorylated $\mathrm{PRD}_{703-868}^{\text {Strep }}$ and its cysteine variants are shown in Figure $6 B$. When the paramagnetic label was located at site $\mathrm{S} 712 \mathrm{C}$ of $\mathrm{PRD}_{703-868}^{\text {Strep }}$, two lone $\geq 25 \mathrm{~s}^{-1}$ PREs were observed (residues 233 and 336 of Bro1), whereas no strong PRE of $\geq 25 \mathrm{~s}^{-1}$ were seen when the label was conjugated to site A756C of PRD ${ }_{703-868}^{\text {Strep }}$. These minimal intermolecular PRE effects indicate that interactions of Bro1 with sites S712C and A756C of PRD Pro3-868 $^{\text {Stre }}$ are negligible. These observations validate the results of our NMR titration experiments, which demonstrated that the isolated Brol did not associate with the $\mathrm{N}$-terminal portion of PRD and rule out transient association between the highly basic region of PRD encompassing site A756C and electronegative surface accessible regions of Bro1. When the paramagnetic label was located at site C813 of $\mathrm{PRD}_{703-868}^{\text {Strep }}$, strong PREs $\left(\geq 25 \mathrm{~s}^{-1}\right)$ were observed for several Bro1 residues, with residues 91, 114, 116, and 233 completely 

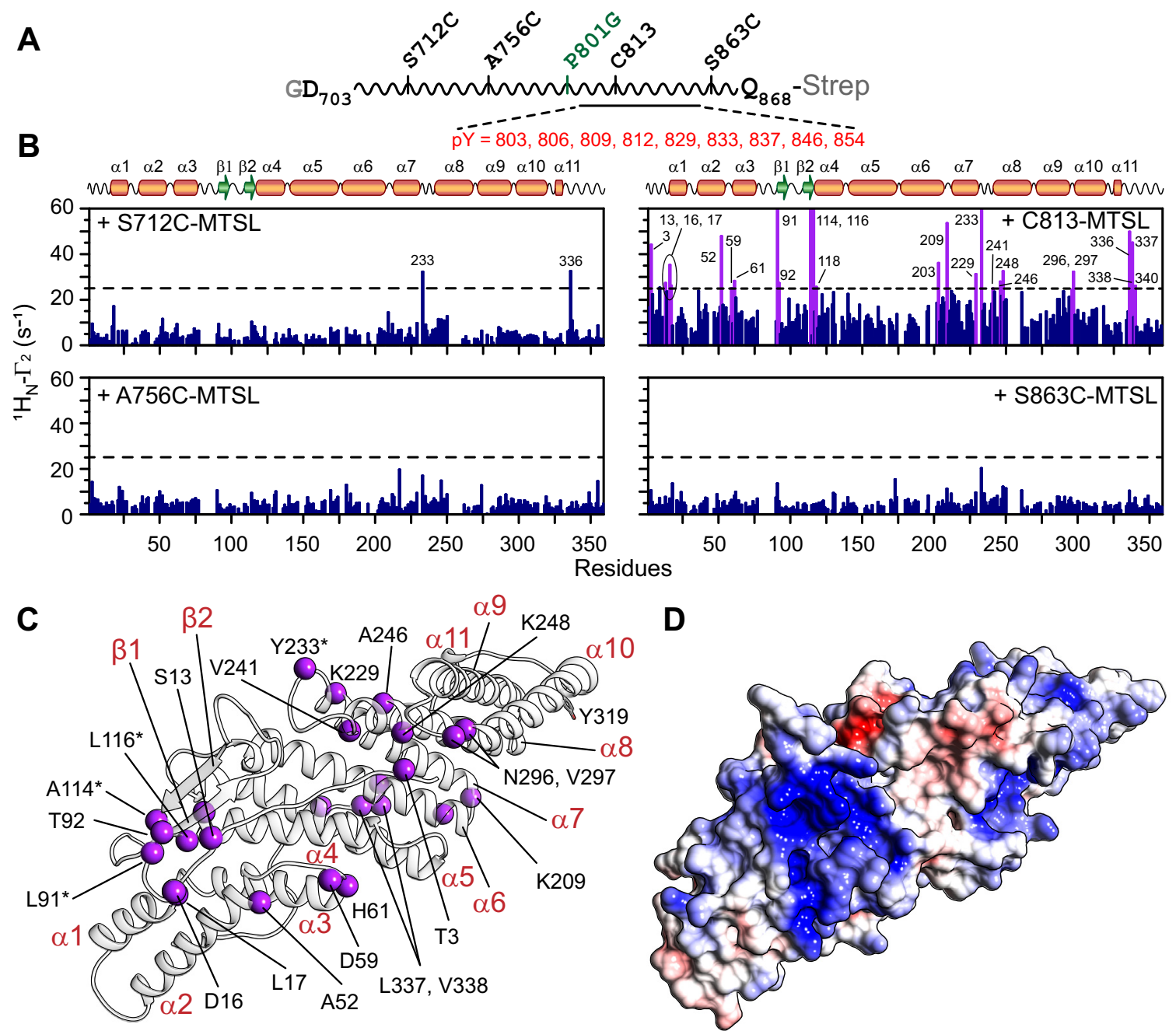

Figure 6. PRE mapping of Bro1-hyperphosphorylated PRD Strep $_{768}$ interactions. $A$, schematic of $\mathrm{PRD}_{703-868}^{\text {Strep }}$ vertical lines indicate the location of the four independent conjugation sites and of the P801G mutation (green). The location of phosphorylated tyrosine residues is shown. $B$, intermolecular PREs observed on ${ }^{2} \mathrm{H} /{ }^{15} \mathrm{~N}$-labeled Bro1 $(200 \mu \mathrm{M})$ arising from MTSL label conjugated to hyperphosphorylated $\mathrm{PRD}_{703-868}^{\text {Strep }}$ at four specific sites; Bro1 to PRD ${ }_{703-868}^{\text {Strep }}$ molar ratio $=1: 1$. Conjugation of MTSL at site C813 produced a noticeable intermolecular PRE; residues with intermolecular PREs above the background are labeled and indicated by purple bars. PREs too large $\left(>60 \mathrm{~s}^{-1}\right)$ to be accurately measured are plotted as $60 \mathrm{~s}^{-1}$. The remaining three conjugation sites did not generate large intermolecular PREs; a few exceptions are labeled. C, ribbon representation of Bro1; residues that exhibit large PREs ( $\geq 25 \mathrm{~s}^{-1}$ ) with spin label at site C813 are depicted as purple spheres; residues that were completely broadened out are marked with asterisks. Y319 is shown in a stick representation. $D$, molecular surface of Bro1 (in the same orientation as $C$ ) color coded according to electrostatic potential; $\pm 5 \mathrm{kT}$ with blue, positive; white, neutral; and red, negative. PRD, proline-rich domain; PRE, paramagnetic relaxation enhancement; MTSL, (1-oxyl-2,2,5,5-tetramethyl- $\Delta 3$-pyrroline-3-methyl) methanethiosulfonate.

broadened out. These observations indicate that the spin label is in the close vicinity of the aforementioned Bro1 residues, possibly because of its proximity to phosphorylated tyrosine residues of $\mathrm{PRD}_{703-868}^{\text {Strep }}$ that likely drive the interdomain electrostatic association. No strong PREs $\left(\geq 25 \mathrm{~s}^{-1}\right)$ were observed with the paramagnetic label at site S863C of $\operatorname{PRD}_{703-868}^{\text {Strep }}$, indicating minimal interactions with this site, perhaps because tyrosine residues located next to this site $\left({ }^{864} \mathrm{YY}^{865}\right)$ are not phosphorylated by Src. Control experiments carried out using either free water-soluble paramagnetic nitroxide label, 4hydroxy-2,2,6,6-tetramethylpiperidine 1-oxyl (TEMPOL), or an unrelated MTSL-conjugated protein, apo maltose-binding protein carrying an E38C point mutation (MBP-E38C), showed no significant intermolecular PRE effects (Fig. S9). These results establish that the intermolecular PREs obtained for Bro1 with label at site C813 of hyperphosphorylated $\mathrm{PRD}_{703-868}^{\text {Strep }}$ arise from a specific association of this site with Bro1 and not from preferential binding of the paramagnetic label to Bro1.

A quantitative interpretation of the strong intermolecular PREs obtained with paramagnetic label conjugated to site C813 is not plausible owing to the disordered nature of hyperphosphorylated $\mathrm{PRD}_{703-868}^{\text {Strep }}$ and the complexity of phosphorylation pattern ( $c f$., Fig. $S 8 B$ ), culminating in a highly dynamic encounter complex with Bro1. The experimental manifestation of this dynamic complex is the approximately uniform and elevated ${ }^{1} \mathrm{H}-\Gamma_{2}$ PRE background of $\sim 10$ to $15 \mathrm{~s}^{-1}$ (Fig. $6 B$; for the other three sites of $\mathrm{PRD}_{703-868}^{\text {Strep }}$, this background is relatively lower, $\sim 5-10 \mathrm{~s}^{-1}$ and stems from random collisions of these three sites with surface accessible sites of 


\section{Structure-function studies of tyrosine phosphorylation of ALIX}

Bro1). Mapping these substantial PREs on to the crystal structure of Bro1, however, allowed us to identify the preferential mode of interactions between hyperphosphorylated $\mathrm{PRD}_{703-868}^{\text {Strep }}$ and Bro1. Two clusters of intermolecular contacts can be discerned (Fig. $6 \mathrm{C}$ ), both residing on the two arms of the convex face of Bro1. For comparison, the molecular surface of Bro1 color coded according to electrostatic potential calculated using APBS software (Adaptive Poisson-Boltzmann Solver) (28) is shown in Figure $6 D$. One contact site comprises the N-terminal portion of Bro1, residues 13, 16, 17, 52, 59, 61, $91,92,114,116$, and 118. Because three of these residues were completely broadened out in the presence of paramagnetically labeled hyperphosphorylated $\mathrm{PRD}_{703-868}^{\text {Strep }}$, we speculate that this cluster makes primary contact with paramagnetically labeled site C813. This cluster has a strong basic character and, therefore, can electrostatically associate with hyperphosphorylated $\mathrm{PRD}_{703-868}^{\mathrm{Strep}}$, further validating that the interdomain interactions are governed by phosphorylated tyrosine residues of PRD. The other contact site comprises residues 203, 209, 229, 233, 241, 246, 248, 296, and 297, and residue 3, which lies in close vicinity of this cluster. Because of a less pronounced electropositive surface, we hypothesize that this Bro1 cluster serves as a secondary contact site with hyperphosphorylated PRD $_{703-868}^{\text {Strep }}$. Technical difficulties prevented us from measuring intermolecular PREs on phosphorylated Bro1 owing to a significant precipitation observed upon mixing phosphorylated Bro1 with paramagnetically labeled hyperphosphorylated $\mathrm{PRD}_{703-868}^{\text {Strep }}$. However, since Y319 of Bro1 is located away from the two Bro1 regions that interact with hyperphosphorylated $\mathrm{PRD}_{703-868}^{\text {Strep }}$ (Fig. 6 C), we predict that Bro1 phosphorylation will have a negligible effect on the association of these two domains. Collectively, the aforementioned observations establish that site C813 of hyperphosphorylated $\mathrm{PRD}_{703-868}^{\text {Strep }}$, which lies adjacent to a group of phosphorylated tyrosine residues, transiently interacts with two electropositive regions of Bro1.

\section{Interactions between Bro1 and analogs of late endosomal membranes}

To identify the phospholipid-binding region(s) of Bro1, we made use of NMR titration experiments, where unilamellar lipid vesicles were mixed with NMR-visible Brol. To mimic the composition of late endosomal membranes, extruded vesicles were made using 1-palmitoyl-2-oleoyl-glycero-3phosphocholine (POPC), 1-palmitoyl-2-oleoyl-sn-glycero-3phosphoethanolamine (POPE), and LBPA. We also made use of zwitterionic vesicles comprising POPC and POPE and negatively charged vesicles composed of POPC, POPE, and 1palmitoyl-2-oleoyl-sn-glycero-3-phospho-L-serine (POPS). Note that eukaryotic membranes, including those of late endosomes, are highly complex and that the vesicles used here represent an artificial system. However, the latter offer the most native-like local membrane environment and, therefore, are often considered simplified models of cellular membranes (29). The size distribution of vesicles, in the absence and presence of Bro1, was measured by DLS and found to be homogenous with mean diameters ranging from 130 to $150 \mathrm{~nm}$ (Fig. S10). A previous study suggested that the binding of divalent calcium to Bro1 was necessary for Bro1-membrane interactions (11). However, negligible chemical shift changes were observed for Bro1 resonances in the presence of a molar excess of calcium, which ruled out Bro1-calcium interactions (Fig. S11).

On addition of POPC/POPE/LBPA vesicles (molar ratio of 6:3:1) to $100 \mu \mathrm{M}$ NMR-visible Bro1 (protein to lipid molar ratio: 1:30), $\sim 20$ to $30 \%$ resonance broadening was observed for a majority of ${ }^{1} \mathrm{H}-{ }^{15} \mathrm{~N}$ cross peaks of Brol (Fig. S12A; no chemical shift perturbations and new resonances were observed upon addition of vesicles to Bro1). When LBPA concentration was increased in a stepwise fashion $(10,12.5$, and $15 \mathrm{~mol} \%$ of LBPA; Fig. S12, $A-C$ ), crosspeaks of Bro1 were progressively attenuated. These results indicate the formation of a high-molecular weight complex between Brol and lipid vesicles and an increase in the vesicle-bound population of Bro1 as a function of LBPA concentration; all NMR measurements were carried out using the same protein to lipid molar ratio of 1:30. The reductions in ${ }^{1} \mathrm{H}-{ }^{15} \mathrm{~N}$ cross-peak volumes of Bro1 as a function of increasing LBPA concentration (Bro1 + POPC/POPE/LBPA vesicles, molar ratio 5.5:3:1.5 versus Bro1 + POPC/POPE/LBPA vesicles, molar ratio 6:3:1) are plotted in Figure $7 A$, which permitted identification of the most affected residues that likely associate directly with LBPA-enriched particles. The solvent-exposed motif, residues ${ }^{101}{ }^{K G S L F G G S V K}{ }^{110}$, of the extended loop that connects the two $\beta$-strands of Bro1 was predicted to insert into LBPA-enriched membranes (11). Although reduction of cross-peak volumes was observed for residues 104 to 106, the other residues of this exposed motif were comparatively less affected in the presence of lipid particles, with G107 being the least affected residue (Figs. $7 A$ and S12). A quantitative analysis of the attenuation of ${ }^{1} \mathrm{H}-{ }^{15} \mathrm{~N}$ cross-peak volumes of G107 as a function of increasing LBPA concentration revealed the ratios of $\sim 1$ and $\sim 0.8$ between 12.5 versus 10 and 15 versus $10 \mathrm{~mol} \%$ of LBPA, respectively ( $20 \%$ attenuation; Fig. $7 B$ ). On the other hand, the most affected Bro1 residues, S13 and G55, exhibited $\sim 60 \%$ attenuation in their cross-peak volumes as a function of increasing LBPA concentration. These observations rule out the insertion of this exposed loop into the vesicles since in the event of such insertion, the corresponding residues would exhibit a significantly greater resonance line broadening. Mapping of the most affected Bro1 residues in the presence of LBPA-enriched particles onto the crystal structure of Bro1 revealed two clusters, localized on two arms of the convex face of Bro1, which likely make direct contacts with lipid particles (Fig. 7, $C$ and $D$ ). Cluster 1 comprised residues $10,13,14,17,54,55,98,184$, and 185 , whereas residues 6,239 , 241, 250, 279, 283, 290, 301, 329, and 322 formed cluster 2. Residues 27 to 28 and two extreme C-terminal residues, 352 to 353, were outliers, likely because of nonspecific association with the lipid particles. Y319 of Bro1 is located away from both clusters that interact with lipid particles. A near-perfect match was observed with the pattern and the magnitude of attenuation of resonances of nonphosphorylated and phosphorylated Bro1 (one at a time) in the presence of LPBA-enriched 

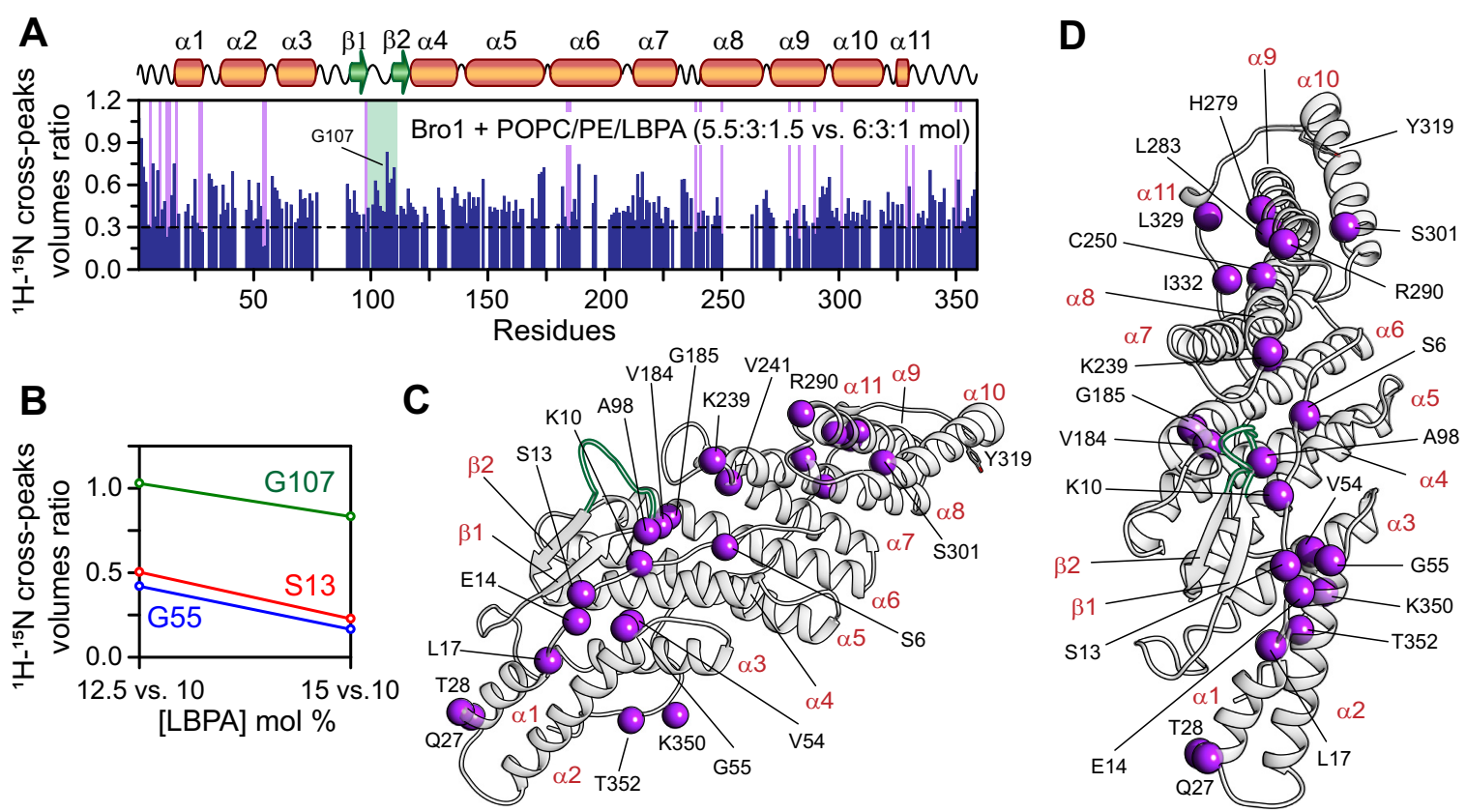

Figure 7. Interaction of Bro1 with LBPA enriched lipid vesicles. $A$, reduction in cross-peak volumes of ${ }^{2} \mathrm{H} /{ }^{15} \mathrm{~N}$-labeled Bro1 (100 $\left.\mu \mathrm{M}\right)$ as a function of increasing amount of LBPA; Bro1 + POPC/POPE/LBPA vesicles (5.5:3:1.5 versus 6:3:1), the numbers in parenthesis represent the molar ratio of each phospholipid in vesicles. Bro1 to lipid molar ratio $=1: 30$. Semitransparent purple rectangles indicate residues that exhibit significant attenuation $(\leq 0.3)$ in the presence of increasing concentrations of LBPA. A semitransparent green rectangle marks the location of the flexible loop connecting the two $\beta$-strands; G107 is marked. $B$, relative changes in cross-peak volumes of three representative residues as a function of increasing concentration of LBPA. The ratios of crosspeak volumes were obtained by recording three ${ }^{1} \mathrm{H}-{ }^{15} \mathrm{~N}$ TROSY-HSQC spectra of Bro1 in the presence of vesicles containing increasing amounts of LBPA. The following vesicle compositions were used: POPC/POPE/LBPA molar ratios 6:3:1, 5.75:3:1.25, and 5.5:3:1.5. $C$ and $D$, ribbon representation of Bro1 in two different orientations; the most affected residues upon association with LBPA-enriched vesicles are depicted as purple spheres. Y319 is shown in a stick representation. The extended loop connecting the two $\beta$-strands is marked in green. HSQC, heteronuclear single quantum coherence; LBPA, 2,2' lysobisphosphatidic acid; POPC, 1-palmitoyl-2-oleoyl-glycero-3-phosphocholine; POPE, 1-palmitoyl-2-oleoyl-sn-glycero-3-phosphoethanolamine; TROSY, transverse relaxation optimized spectroscopy.

particles, indicating that Src-mediated phosphorylation of Bro1 plays no major role in interactions with phospholipids (Fig. S13). A close agreement between the two clusters that interact with lipid vesicles and the electropositive regions of Bro1 (cf., Figs. $6 C$ and 7, $C$ and $D$ ) indicated that interactions between Bro1 and lipid particles were primarily governed by the negative charge of LBPA. To confirm the electrostatic nature of these interactions, similar titration experiments were carried out using Bro1, in the absence and presence of zwitterionic and negatively charged vesicles (POPC/POPE and POPC/POPE/POPS, one at a time, Fig. S14, $A$ and $B$, respectively). Negligible signal reductions were observed with zwitterionic vesicles (POPC/POPE molar ratio 7:3). These results also rule out the role of viscosity on attenuation of Bro1 resonances in the presence of vesicles. Negatively charged POPC/ POPE/POPS particles (molar ratio of 5.5:3:1.5) produced a very similar attenuation profile as that of Bro1 + POPC/POPE/ LBPA vesicles, indicating that ionic interactions drive the association between Brol and lipid particles. Finally, we note that despite similarities, the magnitude of cross-peak attenuation is greater with LBPA than POPS particles ( $c f$., Figs. S12C and $S 14 B$, respectively), indicating a stronger association of Bro1 with LBPA-enriched particles.

\section{Discussion}

In summary, we establish that tyrosine phosphorylation of ALIX-PRD plays a critical role in its polymerization and that hyperphosphorylated PRD likely competes with anionic phospholipids for binding to the Brol domain. These data elucidate the underlying mechanism by which tyrosine phosphorylation triggers the cellular redistribution of ALIX and its impact on intramolecular and intermolecular associations that dictate the broad functional repertoire of ALIX in cell signaling.

$\mathrm{PRD}_{703-868}^{\text {Strep }}$ exhibited remarkable aggregation properties and formed $\beta$-sheet-rich amyloid fibrils that dissolved on Src kinase-mediated phosphorylation and reassembled on PTP1B-mediated dephosphorylation of its conserved tyrosine residues. LC-MS/MS analyses of PK digestion of $\mathrm{PRD}_{703-868}^{\text {Strep }}$ fibrils revealed two protected fragments, residues 800 to 813 and 840 to 863 , that likely form the fibril core. The motif encompassing residues 800 to 813 of PRD encodes epitopes of two binding partners, centrosomal protein of $55 \mathrm{kDa}$ (CEP55), which is a mitotic phosphoprotein involved in cytokinetic abscission (30), and apoptosis linked gene-2 (ALG-2), which is a calcium-binding protein necessary for cell death (31). We speculate that the formation of ALIX fibrils provides a stringent regulatory control as the binding epitopes required for the recruitment of CEP55 and ALG-2 will be unavailable because of their involvement in fibril formation, whereas Src-mediated disassembly of ALIX fibrils will allow for the subsequent recruitment of CEP55 and ALG-2 by ALIX. In line with these hypotheses, a cellular study uncovered that inhibition of the focal adhesion kinase-Src signaling pathway affected the 


\section{Structure-function studies of tyrosine phosphorylation of ALIX}

timing of CEP55 recruitment at the midbody and blocked cytokinetic abscission (32). Src-mediated hyperphosphorylation of PRD results in a heterogenous phosphorylation pattern in vitro. This complexity may provide additional regulatory control for ALIX-mediated ALG2 recruitment and subsequent cell death. This is because the tyrosine residues of the PRD motif, residues 800 to 813 , were shown to be critical for ALG2 association (33). The C-terminal residues of PRD (residues 832-868, especially motif ${ }^{852}$ PSYP $^{855}$ ) were shown to be essential for ALIX multimerization in vivo (30). These observations are consistent with our in vitro findings that residues 840 to 863 of PRD are involved in fibril formation. The filament-forming components of the endosomal sorting complexes required for transport pathway known as charged multivesicular body proteins (CHMPs) recruit ALIX through its Bro1 domain to endosomal membranes (34, 35). Conversely, ALIX-Bro1 recruits and nucleates CHMPs (36), which are the main drivers of endosomal sorting complexes required for transport-mediated membrane remodeling. We predict that the formation of PRD fibrils will likely result in a multiplicative increase in the binding affinity between Bro1 and CHMPs, culminating in nucleation and polymerization of CHMPs. These observations make a strong case for the existence of PRD-mediated ALIX assemblies in vivo and suggest a vital role of PTMs in regulating the timing of the recruitment of signaling partners of ALIX.

The structural characteristics and dynamics of the Bro1 domain were assessed using NMR spectroscopy. Conformational changes stemming from Src-mediated phosphorylation of residue Y319 of Bro1 were found to be localized around this tyrosine. NMR titration experiments ruled out the interdomain association between $\mathrm{PRD}_{703-800}^{\text {Strep }}$ and Bro1. These results are contradictory to a previous study, which indicated that the N-terminal portion of the PRD of intact ALIX associates with the Brol domain and inhibits its interaction with CHMPs (13). Our results agree with a previous biophysical study that used full-length ALIX produced in insect cells and found that the presence of PRD did not influence the interactions between intact ALIX and peptide analogs of CHMPs (14). The discrepancy between these two studies was attributed to the use of pure monomeric ALIX in the biophysical investigation (14) versus the use of crude extracts in the cellular study (13) and the likely involvement of additional factors such as avidity effects because of ALIX and CHMP oligomerization influencing their association. Because highly sensitive PRE experiments also ruled out the association between Bro1 and the N-terminal portion of PRD at sites S712C and A756C, we argue that the cellular results of intramolecular association between the N-terminal portion of PRD and Brol and the corresponding prevention of Bro1 binding to CHMPs (13) could have been influenced by other adapter proteins present in crude cell extracts. Note that fibril formation was not observed in full-length ALIX made using insect cells (14), likely because of potential unaccounted PTMs such as hyperphosphorylation of PRD or because the globular domains of ALIX increase the critical concentration required for its fibrillization.
Paramagnetic NMR measurements revealed transient electrostatic interactions between Brol and hyperphosphorylated PRD. In intact ALIX, these interdomain interactions will likely be substantially stronger owing to their intramolecular nature. These interactions stem from a specific association between site C813 of hyperphosphorylated PRD and the basic surface of Bro1 and are governed by a patch of phosphorylated tyrosine residues encompassing residue C813. Since the same motif, residues 800 to 813 , is likely involved in fibril formation, these intramolecular interactions will not be plausible in PRDmediated ALIX assemblies. The biophysical study mentioned previously uncovered that the presence of PRD results in inhibition of the binding of the $\mathrm{V}$ domain to $\mathrm{YPX}_{\mathrm{n}} \mathrm{L}$ motifs in monomeric full-length ALIX (14). Although NMR experiments rule out the association between the $\mathrm{N}$-terminal portion of PRD and the V domain, interdomain association between Bro1 and hyperphosphorylated PRD in full-length ALIX can likely interfere with the interactions of the $\mathrm{V}$ domain with its binding partners. NMR titration experiments between Bro1 and LBPA-enriched lipid vesicles revealed that these electrostatic interactions localized on the same basic surface of Bro1 that comes into direct contact with hyperphosphorylated PRD. Competition between intramolecular and intermolecular interactions is, thus, likely to redistribute ALIX away from late endosomal membranes to the cytosol. These observations are consistent with a recent in vivo study that demonstrated that deletion of PRD facilitated ALIX membrane association (37). Phosphorylation of Bro1 neither was found to play a major role in interactions between Bro1 and anionic lipid vesicles nor is it likely involved in the interdomain interactions with hyperphosphorylated PRD. Even though the Src-binding motif represents the most conserved set of residues in mammalian Bro1 domains, mutation of Y319 of the yeast Bro1 ortholog had no effect on multivesicular body sorting (38). Our results are consistent with this observation and indicate that the phosphorylation of this residue is linked to other functions in mammalian cells that do not involve membrane associations.

Altogether, the aforementioned results indicate that the PTM-mediated formation and dissolution of ALIX amyloids will provide a strict spatiotemporal control on the recruitment of its binding partners involved in cytokinesis and apoptosis and will likely control nucleation of CHMPs and the corresponding endosomal membrane remodeling. ALIX-Src interactions at late endosomal membranes will result in phosphorylation of Bro1 and PRD of ALIX and shift the equilibrium toward monomeric ALIX. The hyperphosphorylated PRD will then compete against late endosomal membranes for binding to Bro1, culminating in a redistribution of ALIX back to the cytosol. Finally, we note that amyloids are commonly associated with proteinopathies, including Alzheimer's and Parkinson's diseases (39). Recent discoveries of functional amyloids have challenged this perception by elucidating the physiological roles of amyloids and their ubiquitous distribution in lower order and higher order organisms (40-43). These discoveries have led to the emergence of a new narrative, which posits that amyloidogenesis represents a functional phase transition, similar to the one found in 


\section{Structure-function studies of tyrosine phosphorylation of ALIX}

nature between gas, liquid, and solid phases, and suggests that cells may harness many useful characteristics of amyloids, including extreme stability, avidity, prion-like replication, and many others. Exactly how cells generate physiological amyloids without succumbing to pathology is not known. One suggested mechanism is the dissolution of amyloids, often through chaperones or changes in $\mathrm{pH}$. The modulation of amyloids via PTMs described here, specifically a rapid disassembly and assembly of fibrils through tyrosine phosphorylation and dephosphorylation, respectively, represents another elegant mechanism with which cells may control the associated cytotoxicity. Given the prevalence of phosphorylation in eukaryotes (44) and that many proteins are prone to aggregation (45, 46), we hypothesize that such regulatory control on amyloid production is widespread, allowing eukaryotic cells to escape the cytotoxic effects of functional amyloids.

\section{Experimental procedures}

\section{Materials}

IPTG and arabinose were purchased from Sigma-Aldrich (catalog no. 420322 and A3256, respectively). ThT was purchased from Thermo Fisher Scientific (catalog no. AC211760050). CR was purchased from Sigma-Aldrich (catalog no. C6277). Buffered ATP solution ( $\mathrm{pH}$ 7.5) was obtained from Thermo Fisher Scientific (catalog no. R1441). Phosphotyrosine mouse monoclonal antibody was obtained from Cell Signaling Technology (catalog no. 9411). Secondary antibody, goat antimouse IgG, was obtained from Thermo Fisher Scientific (catalog no. G-21040). The malachite green phosphate detection kit and PTP1B substrate were purchased from R\&D Systems, Inc (catalog no. DY996 and ES006, respectively). PK solution $(20 \mathrm{mg} / \mathrm{ml})$ was purchased from Thermo Fisher Scientific (catalog no. 25530049). Reagents for NMR isotopic enrichment were obtained from Cambridge Isotopes Laboratories (CIL) and Sigma-Aldrich. Paramagnetic MTSL and its diamagnetic counterpart, (1-acetoxy-2,2,5,5-tetramethyl- $\delta$-3pyrroline-3-methyl) methanethiosulfonate (MTS), were purchased from Toronto Research Chemicals, Inc (catalog no. O875000 and A167900, respectively). TEMPOL was purchased from Sigma-Aldrich (catalog no. 176141). Phospholipids, POPC, POPE, and POPS were purchased from Avanti Polar Lipids, Inc (catalog no. 850457C, 850757C, 840034C, respectively). LBPA was purchased from Thermo Fisher Scientific (catalog no. 50720538).

\section{Recombinant protein expression and purification}

Codon-optimized (Genewiz, Inc) ALIX-PRD constructs (UniProt accession no. Q8WUM4), GB1 - PRD $_{703-868}^{\text {Strep }}$ and GB1 - PRD ${ }_{703-800}^{\text {Strep }}$, were subcloned in pET11a (Novagen, EMD Millipore) and expressed in BL21(DE3) competent cells (Agilent; catalog no. 200131). Cysteine variants of GB1 - PRD ${ }_{703-868}^{\text {Stre }}$ used for paramagnetic NMR measurements were generated using site-directed mutagenesis. The recombinant constructs representing globular ALIX domains, namely Bro1 (residues 1-359), V (residues 359-702), and Bro1-V (residues 1-702), were obtained from Addgene, accession no. 80641 (47), 17639 (48), and 42577 (49), respectively. The human Src kinase construct (UniProt accession no. P12931) comprising a C-terminal tobacco etch virus (TEV)cleavable polyhistidine tag, pEX-Src-C-His, was a generous gift from the van der Vliet Group (University of Vermont) and was expressed in BL21-AI cells (Thermo Fisher Scientific; catalog no. C607003). The construct representing the catalytic domain of human PTP1B (residues 1-301; UniProt accession no. P18031) was obtained from Addgene, accession no. 102719 (50) and expressed in BL21(DE3) competent cells. The TEV protease construct was a generous gift from David S. Waugh (National Cancer Institute, the National Institutes of Health $[\mathrm{NIH}]$ ). The construct of MBP-E38C (UniProt accession no. P0AEX9) was a generous gift from G. Marius Clore (National Institute of Diabetes and Digestive and Kidney Diseases, the $\mathrm{NIH}$ ). MBP-E38C was expressed in BL21-CodonPlus (DE3)RIPL competent cells (Agilent; catalog no. 230280).

ALIX-PRD constructs, GB1 - $\mathrm{PRD}_{703-868}^{\text {Strep }}$ and its cysteine variants and $\mathrm{GB1}-\mathrm{PRD}_{703-800}^{\text {Strep }}$ were expressed at $16{ }^{\circ} \mathrm{C}$. Briefly, cells were grown at $37^{\circ} \mathrm{C}$ in $1 \mathrm{~L} \mathrm{LB}$ medium (MP Biomedicals; catalog no. 3002-036) at natural isotopic abundance or minimal M9 medium for isotopic labeling. The latter contained $1 \mathrm{~g} / 1{ }^{15} \mathrm{NH}_{4} \mathrm{Cl}(\mathrm{CIL})$ for ${ }^{15} \mathrm{~N}$ labeling. About $30 \mathrm{~min}$ prior to induction, the temperature of cell culture was reduced to $16{ }^{\circ} \mathrm{C}$. Cells were induced with $1 \mathrm{mM}$ IPTG at an absorbance of $\sim 0.8$ at $600 \mathrm{~nm}$ and harvested after $\sim 48 \mathrm{~h}$. Src kinase was expressed according to our previous protocol (16). Briefly, cells were grown at $37{ }^{\circ} \mathrm{C}$ in $1 \mathrm{~L}$ terrific broth medium (Thermo Fisher Scientific; catalog no. BP9728) at natural isotopic abundance. About 30 min prior to induction, the temperature of cell culture was reduced to $16{ }^{\circ} \mathrm{C}$. Cells were induced with $0.2 \% \mathrm{w} / \mathrm{v}$ arabinose and $1 \mathrm{mM}$ IPTG at an absorbance of $\sim 0.8$ at $600 \mathrm{~nm}$ and harvested $\sim 24 \mathrm{~h}$ after induction. The catalytic domain of PTP1B was expressed at 16 ${ }^{\circ} \mathrm{C}$. Cells were grown at $37^{\circ} \mathrm{C}$ in $1 \mathrm{~L} \mathrm{LB}$ medium and $\sim 30 \mathrm{~min}$ prior to induction, and the temperature of cell culture was reduced to $16{ }^{\circ} \mathrm{C}$. Cells were induced with $1 \mathrm{mM}$ IPTG at an absorbance of $\sim 0.8$ at $600 \mathrm{~nm}$ and harvested $\sim 24 \mathrm{~h}$ after induction. For TEV protease, cells were grown at $37^{\circ} \mathrm{C}$ in $1 \mathrm{~L} \mathrm{LB}$ medium and harvested $\sim 4 \mathrm{~h}$ after induction with $1 \mathrm{mM}$ IPTG. The recombinant constructs of globular ALIX domains, namely Bro1, V, and Bro1-V, were expressed at $16{ }^{\circ} \mathrm{C}$. Cells were grown at $37^{\circ} \mathrm{C}$ in $1 \mathrm{~L} \mathrm{LB}$ medium at natural isotopic abundance or in the case of Bro1, in M9 medium for isotopic labeling. The latter contained $0.3 \mathrm{~g} /{ }^{2} \mathrm{H} /{ }^{15} \mathrm{~N} /{ }^{13} \mathrm{C}$ Isogro (Sigma-Aldrich), 99.9\% (v/v) deuterium oxide $\left(\mathrm{D}_{2} \mathrm{O} ; \mathrm{CIL}\right), 1 \mathrm{~g} /$ $1^{15} \mathrm{NH}_{4} \mathrm{Cl}(\mathrm{CIL})$, and $3 \mathrm{~g} / \mathrm{l}^{2} \mathrm{H}_{7},{ }^{13} \mathrm{C}_{6}$-D-glucose (Sigma-Aldrich) for ${ }^{2} \mathrm{H} /{ }^{15} \mathrm{~N} /{ }^{13} \mathrm{C}$ labeling and $0.3 \mathrm{~g} / \mathrm{l}^{2} \mathrm{H} /{ }^{15} \mathrm{~N}$ Isogro (SigmaAldrich), 99.9\% (v/v) deuterium oxide (CIL), $1 \mathrm{~g} / 1{ }^{15} \mathrm{NH}_{4} \mathrm{Cl}$ (CIL), and $3 \mathrm{~g} / \mathrm{l}^{2} \mathrm{H}_{7},{ }^{12} \mathrm{C}_{6}$-D-glucose (CIL) for ${ }^{2} \mathrm{H} /{ }^{15} \mathrm{~N}$ labeling. About $30 \mathrm{~min}$ prior to induction, the temperature of cell culture was reduced to $16{ }^{\circ} \mathrm{C}$. Cells were induced with $1 \mathrm{mM}$ IPTG at an absorbance of $\sim 0.8$ at $600 \mathrm{~nm}$ and harvested after $\sim 24 \mathrm{~h}$.

PRD constructs were purified using a combination of affinity chromatography (ÄKTA Pure and Start Protein Purification Systems; GE Healthcare) and reverse-phase HPLC (1260 


\section{Structure-function studies of tyrosine phosphorylation of ALIX}

Infinity II Liquid Chromatography System; Agilent Technologies). The purification protocol for $\mathrm{GB1}-\mathrm{PRD}_{703-800}^{\text {Strep }}$ is described in our previous work (16). Briefly, the cells were resuspended in a lysis buffer containing $50 \mathrm{mM}$ Tris(hydroxymethyl)aminomethane (Tris), pH 8.0, 2 mM EDTA, and $250 \mathrm{mM} \mathrm{NaCl}$. Cells were lysed by heat shock $\left(80^{\circ} \mathrm{C}, \sim 5 \mathrm{~min}\right)$ and cleared by centrifugation $(48,380 \mathrm{~g}, 25 \mathrm{~min})$. The cell lysate was loaded onto a Streptactin Sepharose column (GE Healthcare), pre-equilibrated with a running buffer containing $50 \mathrm{mM}$ Tris, $\mathrm{pH}$ 8.0, $2 \mathrm{mM}$ EDTA, and $250 \mathrm{mM} \mathrm{NaCl}$, and eluted in the same buffer containing $2.5 \mathrm{mM}$ D-desthiobiotin (Sigma-Aldrich). The eluted GB1 - PRD $_{703-800}^{\text {Strep }}$ protein was mixed with recombinant TEV protease (molar ratio of 50:1) in the presence of $1 \mathrm{mM}$ DTT (Sigma-Aldrich) to hydrolyze the $\mathrm{N}$-terminal GB1 solubility tag. Proteolysis was allowed to proceed at room temperature for $\sim 12 \mathrm{~h}$ and was assessed for completion by SDS-PAGE; Bolt $4-12 \%$ bis-Tris gel (Thermo Fisher Scientific). The hydrolyzed product, $\mathrm{PRD}_{703-800}^{\text {Strep }}$, was further purified using reverse-phase HPLC (Jupiter $10 \mu \mathrm{m}$ C18 $300 \AA$ Å column; Phenomenex, catalog no. 00G-4055-N0) with a 5 to $55 \%$ acetonitrile gradient comprising $0.05 \%$ TFA (SigmaAldrich). The eluted protein was freeze dried (Labconco -84 ${ }^{\circ} \mathrm{C}$ Benchtop Freeze Dryer) and stored at $-80{ }^{\circ} \mathrm{C}$ before use. For GB1 - PRD ${ }_{703-868}^{\text {Strep }}$ and its cysteine variants, the cells were resuspended in a denaturing buffer containing $50 \mathrm{mM}$ Tris, $\mathrm{pH}$ 8.0, and $6 \mathrm{M}$ guanidine hydrochloride (Sigma-Aldrich). Cells were lysed using a homogenizer, EmulsiFlex-C3 (Avestin) and cleared by centrifugation. The cell lysate was filtered through a $0.45 \mu \mathrm{m}$ vacuum-driven filtration device (Stericup; SigmaAldrich) and loaded onto a HisTrap column (GE Healthcare), pre-equilibrated with a denaturing buffer containing $50 \mathrm{mM}$ Tris, $\mathrm{pH} 8.0$, and $6 \mathrm{M}$ guanidine hydrochloride. The bound protein was washed with ten column volumes of refolding buffer containing $50 \mathrm{mM}$ Tris, $\mathrm{pH} 8.0$, and $250 \mathrm{mM} \mathrm{NaCl}$ and eluted in the same buffer containing $0.5 \mathrm{M}$ imidazole (SigmaAldrich). The eluted protein was loaded onto a Streptactin Sepharose column, pre-equilibrated with a running buffer containing $50 \mathrm{mM}$ Tris, $\mathrm{pH} 8.0,2 \mathrm{mM}$ EDTA, and $250 \mathrm{mM}$ $\mathrm{NaCl}$, and eluted in the same buffer containing $2.5 \mathrm{mM}$ Ddesthiobiotin. The resultant elution was mixed with recombinant TEV protease (molar ratio of 100:1) in the presence of $1 \mathrm{mM}$ DTT to hydrolyze the N-terminal GB1 solubility tag. The proteolysis reaction was carried out at room temperature $(\sim 12 \mathrm{~h})$ and assessed for completion by SDS-PAGE. The hydrolyzed product, $\mathrm{PRD}_{703-868}^{\text {Strep }}$, was further purified using reverse-phase HPLC (Jupiter $10 \mu \mathrm{m} \mathrm{C18} 300 \AA \AA$ column) using a 25 to $37 \%$ acetonitrile gradient comprising $0.1 \%$ TFA. The eluted $\mathrm{PRD}_{703-868}^{\text {Strep }}$ fractions were pooled, lyophilized, and stored at $-80{ }^{\circ} \mathrm{C}$.

The purification protocol for Src kinase is described in our previous work (16). Briefly, the cells were resuspended in a lysis buffer containing $50 \mathrm{mM}$ Tris, $\mathrm{pH} 8.0$, and $250 \mathrm{mM} \mathrm{NaCl}$. Cells were lysed using an EmulsiFlex-C3 and cleared by centrifugation. The cell lysate was loaded onto a HisTrap column (GE Healthcare) with a 0 to $1 \mathrm{M}$ imidazole gradient containing $50 \mathrm{mM}$ Tris, $\mathrm{pH} 8.0$, and $250 \mathrm{mM} \mathrm{NaCl}$. The eluted protein was loaded onto a Q Sepharose HP column (GE
Healthcare) with a 0 to $1 \mathrm{M} \mathrm{NaCl}$ gradient in a buffer containing $50 \mathrm{mM}$ Tris, $\mathrm{pH} 8.0$, and $5 \mathrm{mM} \beta$-mercaptoethanol. The eluted fractions were pooled, concentrated (Amicon Ultra-15; cutoff of $30 \mathrm{kDa}$ ), and loaded onto a HiLoad 26/600 Superdex 75 pg column (GE Healthcare) pre-equilibrated with $50 \mathrm{mM}$ Tris, $\mathrm{pH} 7.5,250 \mathrm{mM} \mathrm{NaCl}$, and $5 \mathrm{mM} \beta$-mercaptoethanol. Relevant fractions were pooled and concentrated to $\sim 1 \mathrm{mg} / \mathrm{ml}$ (Amicon Ultra-15; cutoff of $30 \mathrm{kDa}$ ). Samples were aliquoted, flash frozen, and stored at $-80{ }^{\circ} \mathrm{C}$.

For the catalytic domain of PTP1B, the cells were resuspended in a lysis buffer containing $50 \mathrm{mM}$ Tris, $\mathrm{pH} 8.0$, and $250 \mathrm{mM} \mathrm{NaCl}$. Cells were lysed using an EmulsiFlex-C3 and cleared by centrifugation. The cell lysate was loaded onto a HisTrap column (GE Healthcare) with a 0 to $1 \mathrm{M}$ imidazole gradient containing $50 \mathrm{mM}$ Tris, $\mathrm{pH} 8.0$, and $250 \mathrm{mM} \mathrm{NaCl}$. The eluted protein was concentrated (Amicon Ultra-15; cutoff of $10 \mathrm{kDa}$ ) and loaded onto a HiLoad 26/600 Superdex $75 \mathrm{pg}$ column (GE Healthcare) pre-equilibrated with $50 \mathrm{mM}$ Tris, $\mathrm{pH} 8$, and $250 \mathrm{mM} \mathrm{NaCl}$. Relevant fractions were pooled and mixed with recombinant TEV protease (molar ratio of 50:1) in the presence of $1 \mathrm{mM}$ DTT to hydrolyze the $\mathrm{N}$-terminal polyhistidine $(6 \times$ His) affinity tag. The proteolysis reaction was carried out at room temperature $(\sim 12 \mathrm{~h})$ and assessed for completion by SDS-PAGE. The reaction mixture was loaded back onto a HisTrap column (GE Healthcare). The resultant flow-through fractions of hydrolyzed PTP1B were pooled, concentrated, and further purified by size exclusion chromatography (HiLoad 26/600 Superdex 75 pg column; GE Healthcare). Relevant fractions were concentrated $(\sim 3 \mathrm{mg} / \mathrm{ml}$; Amicon Ultra-15; cutoff of $10 \mathrm{kDa}$ ), flash frozen, and stored at $-80^{\circ} \mathrm{C}$.

The purification protocol for TEV protease is described in our previous works $(16,51)$. Briefly, the cells were resuspended in a lysis buffer containing $50 \mathrm{mM}$ Tris, $\mathrm{pH} 8.0$, and $250 \mathrm{mM}$ $\mathrm{NaCl}$. Cells were lysed using an EmulsiFlex-C3 and cleared by centrifugation. The cell lysate was loaded onto a HisTrap column (GE Healthcare) with a 0 to $1 \mathrm{M}$ imidazole gradient containing $50 \mathrm{mM}$ Tris, $\mathrm{pH} 8.0$, and $250 \mathrm{mM} \mathrm{NaCl}$. The eluted protein was concentrated (Amicon Ultra-15; cutoff of $10 \mathrm{kDa}$ ) and loaded onto a HiLoad 26/600 Superdex 75 pg column (GE Healthcare) pre-equilibrated with $50 \mathrm{mM}$ Tris, $\mathrm{pH} 8,250 \mathrm{mM}$ $\mathrm{NaCl}$, and $1 \mathrm{mM}$ DTT. Relevant fractions were pooled and concentrated to $\sim 1 \mathrm{mg} / \mathrm{ml}$ (Amicon Ultra-15; cutoff of 10 $\mathrm{kDa}$ ). The samples were aliquoted, flash frozen, and stored at $-80{ }^{\circ} \mathrm{C}$.

For the Bro1 domain, the cells were resuspended in a lysis buffer containing $50 \mathrm{mM}$ Tris, $\mathrm{pH} 8.0$, and $250 \mathrm{mM} \mathrm{NaCl}$. Cells were lysed using EmulsiFlex-C3 and cleared by centrifugation. The cell lysate was loaded onto a HisTrap column (GE Healthcare) with a 0 to $1 \mathrm{M}$ imidazole gradient containing $50 \mathrm{mM}$ Tris, $\mathrm{pH} 8.0$, and $250 \mathrm{mM} \mathrm{NaCl}$. The eluted protein was concentrated (Amicon Ultra-15; cutoff of $10 \mathrm{kDa}$ ) and loaded onto a HiLoad 26/600 Superdex 75 pg column (GE Healthcare) pre-equilibrated with $50 \mathrm{mM}$ Tris, $\mathrm{pH} 8,250 \mathrm{mM}$ $\mathrm{NaCl}$, and $1 \mathrm{mM}$ DTT. Relevant fractions were pooled and mixed with recombinant TEV protease to cleave off the $\mathrm{N}$-terminal $10 \times$ His affinity tag (molar ratio of 5:1). Proteolysis 


\section{Structure-function studies of tyrosine phosphorylation of ALIX}

was carried out at room temperature $(\sim 48 \mathrm{~h})$ and assessed for completion using SDS-PAGE. The reaction mixture was loaded back onto a HisTrap column (GE Healthcare). The resultant flow-through fractions of hydrolyzed Bro1 were pooled, concentrated, and further purified by size exclusion chromatography (HiLoad 26/600 Superdex 75 pg column; GE Healthcare). Relevant Bro1 fractions were pooled and stored at $-80{ }^{\circ} \mathrm{C}$.

For the $\mathrm{V}$ domain, the cells were resuspended in a lysis buffer containing $50 \mathrm{mM}$ Tris, $\mathrm{pH} 8.0$, and $250 \mathrm{mM} \mathrm{NaCl}$. Cells were lysed using an EmulsiFlex-C3 and cleared by centrifugation. Cell lysate was loaded onto a Glutathione Sepharose 4 Fast Flow column (GE Healthcare) with a 0 to $10 \mathrm{mM}$ L-glutathione (Sigma-Aldrich) gradient containing $50 \mathrm{mM}$ Tris, $\mathrm{pH} 8.0$, and $250 \mathrm{mM} \mathrm{NaCl}$. The eluted protein was concentrated (Amicon Ultra-15, cutoff of $10 \mathrm{kDa}$ ) and loaded onto a HiLoad 26/600 Superdex 75 pg column (GE Healthcare) pre-equilibrated with $50 \mathrm{mM}$ Tris, $\mathrm{pH} 8,250 \mathrm{mM}$ $\mathrm{NaCl}$, and $1 \mathrm{mM}$ DTT. Relevant fractions were pooled and mixed with recombinant TEV protease to cleave off the Nterminal glutathione- $S$-transferase affinity tag (molar ratio of 50:1). Proteolysis was carried out at room temperature $(\sim 12 \mathrm{~h})$ and assessed for completion using SDS-PAGE. The reaction mixture was loaded back onto a Glutathione Sepharose 4 Fast Flow column (GE Healthcare). The resultant flow-through fractions were pooled, concentrated, and further purified by size exclusion chromatography (HiLoad 26/600 Superdex 75 pg column; GE Healthcare). Relevant fractions were pooled, concentrated ( $\sim 10 \mathrm{mg} / \mathrm{ml}$; Amicon Ultra-15; cutoff of $10 \mathrm{kDa})$, and stored at $-80^{\circ} \mathrm{C}$.

For the Bro1-V construct, the cells were resuspended in a lysis buffer containing $50 \mathrm{mM}$ Tris, $\mathrm{pH} 8.0$, and $250 \mathrm{mM} \mathrm{NaCl}$. Cells were lysed using an EmulsiFlex-C3 and cleared by centrifugation. The cell lysate was loaded onto a HisTrap column (GE Healthcare) with a 0 to $1 \mathrm{M}$ imidazole gradient containing $50 \mathrm{mM}$ Tris, $\mathrm{pH} 8.0$, and $250 \mathrm{mM} \mathrm{NaCl}$. The eluted protein was concentrated (Amicon Ultra-15; cutoff of $30 \mathrm{kDa}$ ) and loaded onto a HiLoad 26/600 Superdex 200 pg column (GE Healthcare) pre-equilibrated with $50 \mathrm{mM}$ Tris, $\mathrm{pH} 8$, $250 \mathrm{mM} \mathrm{NaCl}$, and $1 \mathrm{mM}$ DTT. Relevant fractions were pooled and mixed with recombinant TEV protease to cleave off the N-terminal 6x His affinity tag (molar ratio of 5:1). Proteolysis was carried out at room temperature $(\sim 48 \mathrm{~h})$ and assessed for completion using SDS-PAGE. The reaction mixture was loaded back onto the HisTrap column (GE Healthcare). The resultant flow-through fractions were pooled, concentrated, and further purified by size exclusion chromatography (HiLoad 26/600 Superdex 200 pg column; GE Healthcare). Relevant fractions were pooled and stored at $-80{ }^{\circ} \mathrm{C}$.

For apo MBP-E38C, the cells were resuspended in a lysis buffer containing $50 \mathrm{mM}$ Tris, $\mathrm{pH} 8.0$, and $250 \mathrm{mM} \mathrm{NaCl}$. Cells were lysed using an EmulsiFlex-C3 and cleared by centrifugation. Cell lysate was loaded onto an Amylose resin column (New England BioLabs, Inc) with a 0 to $10 \mathrm{mM}$ maltose gradient containing $50 \mathrm{mM}$ Tris, $\mathrm{pH}$ 8.0, $1 \mathrm{mM}$ DTT, and $250 \mathrm{mM} \mathrm{NaCl}$. The eluted protein was concentrated
(Amicon Ultra-15; cutoff of $30 \mathrm{kDa}$ ) and loaded onto a HiLoad 26/600 Superdex 75 pg column (GE Healthcare) preequilibrated with $50 \mathrm{mM}$ Tris, $\mathrm{pH} 8,1 \mathrm{mM}$ DTT, and $250 \mathrm{mM} \mathrm{NaCl}$. Relevant fractions were pooled and concentrated to $\sim 10 \mathrm{mg} / \mathrm{ml}$ (Amicon Ultra-15; cutoff of $30 \mathrm{kDa}$ ). The samples were aliquoted and flash frozen and stored at $-80^{\circ} \mathrm{C}$.

The aforementioned purification schemes resulted in $\geq 99 \%$ pure proteins with the following yields: $\mathrm{PRD}_{703-800}^{\text {Strep }}(\sim 40 \mathrm{mg} / \mathrm{l})$, $\mathrm{PRD}_{703-868}^{\text {Strep }}(\sim 25 \mathrm{mg} / \mathrm{l})$, Src $(\sim 5 \mathrm{mg} / \mathrm{l})$, PTP1B $(\sim 45 \mathrm{mg} / \mathrm{l})$, Brol ( $\sim 50 \mathrm{mg} / \mathrm{l}), \mathrm{V}(\sim 45 \mathrm{mg} / \mathrm{l}), \operatorname{Bro1}-\mathrm{V}(\sim 40 \mathrm{mg} / \mathrm{l})$, and MBPE38C ( 10 mg/l).

\section{MS analyses}

All protein constructs, the end products of in vitro phosphorylation, and site-specific spin labeling reactions (see below) were verified by MS using our previously published protocols $(16,51)$. Briefly, an Agilent 6230 TOF-mass spectrometer with Jet Stream ESI was used for LC-ESI-TOFMS analysis. The Jet Stream ESI source was operated under positive ion mode with the following parameters: $\mathrm{V}_{\text {Cap }}=3500 \mathrm{~V}$, fragmentor voltage $=175 \mathrm{~V}$, drying gas temperature $=325^{\circ} \mathrm{C}$, sheath gas temperature $=325^{\circ} \mathrm{C}$, drying gas flow rate $=10 \mathrm{l} /$ min, sheath gas flow rate $=10 \mathrm{l} / \mathrm{min}$, and nebulizer pressure $=$ $40 \mathrm{psi}$. The chromatographic separation was performed at room temperature on a Phenomenex Aeris Widepore XB-C18 column $(2.1 \mathrm{~mm}$ inner diameter $\times 50 \mathrm{~mm}$ length, $3.6 \mu \mathrm{m}$ particle size). HPLC-grade water and acetonitrile were used as mobile phases A and B, respectively. Each phase also carried $0.1 \%$ TFA. MassHunter software (Agilent) was used for data acquisition and analysis, and MagTran software (Amgen, Inc) was used for mass spectrum deconvolution (52).

\section{PK digestion}

Fibrils of PRD Ptrep $_{7068}^{\text {Sto }}$ and soluble PRD $D_{703-800}^{\text {Strep }}(\sim 40 \mu \mathrm{M}$ each $)$ were incubated with PK $(1 \mu \mathrm{g} / \mathrm{ml})$ in a buffer comprising $50 \mathrm{mM}$ Tris, $\mathrm{pH} 8,1 \mathrm{mM}$ DTT, and $0.5 \mathrm{mM}$ EDTA $\left(37^{\circ} \mathrm{C}\right.$, $\sim 60 \mathrm{~min}$ ). Aliquots were taken at regular intervals and heated at $100{ }^{\circ} \mathrm{C}$ for $\sim 3 \mathrm{~min}$ to inactivate PK. The reactions were assessed using SDS-PAGE, visualized using PageBlue staining solution (Thermo Fisher Scientific; catalog no. 24620). Reactions were also analyzed by LC-MS/MS using our previously published protocol (16). Briefly, LC-MS/MS analysis was carried out by the nanoLC-Orbitrap XL spectrometer. A fused silica capillary LC column (pulled to a tip with a Sutter P-2000 laser capillary puller) was packed with Agilent Zorbax resin (C18; particle size of $5 \mu \mathrm{m}$ ). The inner diameter of the capillary was $100 \mu \mathrm{m}$, and the stationary phase was packed with a pressure device to a length of $70 \mathrm{~mm}$. The column was equilibrated using an Agilent 1100 HPLC pump, solvent $\mathrm{A}=$ $100 \%$ HPLC-grade water with $0.1 \%$ formic acid and solvent $B=$ $100 \%$ acetonitrile with several 10 to $90 \%$ solvent B step gradients. The PK-digested sample of $\mathrm{PRD}_{703-868}^{\text {Strep }}$ fibrils was diluted by a factor of 100 with $2 \%$ acetonitrile and $0.1 \%$ formic acid and loaded on the column by a pressure device $(2.1 \mu \mathrm{l} ; \sim 2$ pmol on column). The LC gradient program was 1 to $34 \%$ solvent B in 66 min, followed by 6 min of $90 \%$ B, followed by 


\section{Structure-function studies of tyrosine phosphorylation of ALIX}

$1 \% \mathrm{~B}$ at $95 \mathrm{~min}$. Data acquisition method parameters were as follows: the capillary LC was positioned in the Thermo nanoelectrospray interface with a $1.55 \mathrm{kV}$ source voltage, $48 \mathrm{~V}$ capillary voltage, $85 \mathrm{~V}$ tube lens, and $165{ }^{\circ} \mathrm{C}$ capillary temperature. For the ion trap, three micro scans and the FT spectra, two micro scans were averaged. There were seven scan events per cycle (6.5 s), one FT scan (resolution of 30,000) from 200 to $1600 \mathrm{~m} / z$, followed by six ion trap, data-dependent collision-induced dissociation MS/MS scans. Dynamic exclusion was enabled with duration of $40 \mathrm{~s}$ and a repeat count of 2. The ion trap collision-induced dissociation scans had an isolation width of $2.0 \mathrm{~m} / z$ and a normalized collision energy of 35. The data analysis method was as follows: peptide sequence matches were found using the OpenMS workflow (53) with the MS-GF + peptide sequence match search algorithm (54). The false discovery rate was set to $5 \%$. The enzyme parameter was set to "Unspecific cleavage." The search parameters used for LC-MS/MS analyses are tabulated in Table S2, whereas Table S3 lists all peptides identified by these analyses.

\section{Tyrosine phosphorylation}

Lyophilized $\mathrm{PRD}_{703-868}^{\text {Strep }}$ was reconstituted in a buffer comprising $50 \mathrm{mM}$ Tris, pH 7.5, $5 \mathrm{mM} \mathrm{MgCl}_{2}, 2 \mathrm{mM}$ DTT, and $0.5 \mathrm{mM}$ EDTA. The resultant $\mathrm{PRD}_{703-868}^{\text {Strep }}$ solution $(50 \mu \mathrm{M})$ was mixed with $1 \mathrm{mM}$ ATP and $\sim 5 \mu \mathrm{M}$ Src. The phosphorylation reaction was allowed to proceed for $\sim 12 \mathrm{~h}$ at $30^{\circ} \mathrm{C}$. Src was then removed from the reaction mixture using a HisTrap column (GE Healthcare) pre-equilibrated with $50 \mathrm{mM}$ Tris, $\mathrm{pH} 8$, and $250 \mathrm{mM} \mathrm{NaCl}$. The resultant flow-through fractions of phosphorylated $\mathrm{PRD}_{703-868}^{\text {Strep }}$ were pooled, and excess ATP/ ADP were removed using a HiPrep 26/10 Desalting column (GE Healthcare) pre-equilibrated with $20 \mathrm{mM}$ sodium phosphate, pH 6.5, $50 \mathrm{mM} \mathrm{NaCl}, 2 \mathrm{mM}$ EDTA, and $1 \mathrm{mM}$ Tris(2carboxyethyl)phosphine (Sigma-Aldrich).

The eluted fractions of Bro1 and Src from a sizing column were mixed; Bro1 to Src molar ratio of $\sim 5: 1$. The resultant mixture was concentrated to a final volume of $\sim 7 \mathrm{ml}$ (Amicon Ultra-15; cutoff of $30 \mathrm{kDa}$ ) and dialyzed against a buffer containing $50 \mathrm{mM}$ Tris, $\mathrm{pH} \mathrm{8,2} \mathrm{mM}$ ATP, $5 \mathrm{mM} \mathrm{MgCl}_{2}, 2 \mathrm{mM}$ DTT, and 0.5 mM EDTA (Slide-A-Lyzer G2 dialysis cassettes; Thermo Fisher Scientific). The reaction was allowed to proceed for $\sim 18$ h at room temperature with one buffer change at a time point of $\sim 4 \mathrm{~h}$ in the dialysis. Phosphorylated Bro1 was purified from the mixture using the procedure mentioned previously. The status of phosphorylation was assessed using LC-ESI-TOFMS, LC-MS/MS, and NMR. For LC-MS/MS, phosphorylated Bro1 was digested using trypsin (Bro1 to trypsin molar ratio of 50:1; incubation time of $\sim 90 \mathrm{~min}$, and room temperature). Hyperphosphorylated $\mathrm{PRD}_{703-868}^{\text {Strep }}$ samples were digested using $\mathrm{PK}$ ( $\mathrm{PRD}_{703-868}^{\text {Strep }}$ to $\mathrm{PK}$ molar ratio of 1000:1; incubation time of $\sim 5 \mathrm{~min}, 37^{\circ} \mathrm{C}$ ). LC-MS/MS analyses were carried out using a similar procedure as the one used to identify products of PK digestion of PRD $\mathrm{Ptrep}_{703}^{\text {Stres }}$ fibrils. Up to two PTMs per peptide were allowed for the search. Phosphorylation of tyrosine was the most prevalent PTM observed (also see Tables S2 and S3). For Bro1, phosphorylation was also assessed using Western blotting. Bro1 + Src reaction mixture separated using electrophoresis was transferred onto a $0.45 \mu \mathrm{m}$ nitrocellulose membrane (Thermo Fisher Scientific; catalog no. LC2001) using a wettransfer Mini Blot Module (Thermo Fisher Scientific; catalog no. B1000). Procedures for blotting and subsequent product visualization are described previously (16).

\section{Large unilamellar lipid vesicles}

Lipid vesicles were prepared according to the protocols published by Jiang et al. (55). Briefly, lipids were stored in chloroform/methanol $(2: 1)$ stock solutions at $-20{ }^{\circ} \mathrm{C}$. Lipid films were prepared by drying the appropriate amounts of stock solutions under a stream of dry nitrogen followed by vacuum desiccation (vacuum oven; VWR) at room temperature for $\sim 2 \mathrm{~h}$ to remove traces of organic solvents. The resultant lipid films were hydrated using appropriate volumes of buffer containing $20 \mathrm{mM}$ sodium phosphate, $\mathrm{pH} 6.5,50 \mathrm{mM}$ $\mathrm{NaCl}, 1 \mathrm{mM}$ EDTA, and $1 \mathrm{mM}$ TCEP and subjected to vortex mixing (approximately three times, $60 \mathrm{~s}$ each). The multilamellar vesicle solutions were extruded through a $100 \mathrm{~nm}$ diameter polycarbonate membrane (Whatman, GE Healthcare) using an extrusion kit (Avanti Polar Lipids, Inc) and used immediately for NMR and size measurements.

\section{DLS}

The size distribution of lipid vesicles was determined by DLS at $25{ }^{\circ} \mathrm{C}$ using $25 \mu \mathrm{M}$ vesicles, with and without $25 \mu \mathrm{M}$ Bro1. Measurements were performed using the Zetasizer Nano ZS (Malvern Instruments) instrument operating at a wavelength of $633 \mathrm{~nm}$. The measurements were repeated 15 times after a $1 \mathrm{~min}$ temperature equilibration. A cumulant fit and a sphere model were used to obtain the average hydrodynamic radius $(130-150 \mathrm{~nm})$.

\section{Site-specific spin labeling}

MTSL and MTS were reconstituted in ethanol (SigmaAldrich) to a final concentration of $\sim 100 \mathrm{mM}$ and mixed with the protein of interest at a molar ratio of 10:1 (spin label versus protein). The spin labeling reaction was allowed to proceed in the dark for $\sim 12 \mathrm{~h}$ at room temperature and assessed for completion using LC-ESI-TOFMS. The excess of unreacted spin label was removed using the HiPrep 26/10 Desalting column (GE Healthcare) pre-equilibrated with $20 \mathrm{mM}$ sodium phosphate, $\mathrm{pH} 6.5,50 \mathrm{mM} \mathrm{NaCl}$, and $2 \mathrm{mM}$ EDTA. The resultant protein fractions were pooled, concentrated, and immediately used for NMR measurements.

\section{NMR sample preparation}

Samples of ${ }^{15} \mathrm{~N}$-labeled $\mathrm{PRD}_{703-800}^{\text {Strep }},{ }^{15} \mathrm{~N}$-labeled hyperphosphorylated $\mathrm{PRD}_{703-868}^{\text {Strep }}$, and ${ }^{15} \mathrm{~N} /{ }^{2} \mathrm{H}-$ or ${ }^{15} \mathrm{~N} /{ }^{13} \mathrm{C} /{ }^{2} \mathrm{H}$ labeled Bro1 were prepared in a buffer comprising $20 \mathrm{mM}$ sodium phosphate, $\mathrm{pH}$ 6.5, $50 \mathrm{mM} \mathrm{NaCl}, 2 \mathrm{mM}$ EDTA, and $1 \mathrm{mM}$ TCEP. For paramagnetic NMR, the samples were prepared in an identical buffer without TCEP. To assess the interactions between calcium and Bro1, samples were 


\section{Structure-function studies of tyrosine phosphorylation of ALIX}

prepared using $20 \mathrm{mM}$ MES, pH 6.5, $50 \mathrm{mM} \mathrm{NaCl}, 2 \mathrm{mM}$ EDTA, and $1 \mathrm{mM}$ TCEP. All NMR samples contained 7\% v/v $\mathrm{D}_{2} \mathrm{O}$.

\section{NMR spectroscopy}

All NMR experiments were carried out at $30{ }^{\circ} \mathrm{C}$ on Bruker 600 and $800 \mathrm{MHz}$ spectrometers equipped with $z$-gradient triple resonance cryoprobes. Spectra were processed using NMRPipe (56) and analyzed using the CCPN software suite (57). Sequential ${ }^{1} \mathrm{H},{ }^{15} \mathrm{~N}$, and ${ }^{13} \mathrm{C}$ backbone resonance assignments of nonphosphorylated and phosphorylated Bro1 samples were carried out using conventional TROSY-based three-dimensional triple resonance experiments (58). TROSY-based ${ }^{15} \mathrm{~N}\left\{{ }^{1} \mathrm{H}\right\}$ NOE measurements (59) were carried out on ${ }^{2} \mathrm{H} /{ }^{15} \mathrm{~N}$-labeled nonphosphorylated and phosphorylated Brol samples at $800 \mathrm{MHz}$. The ${ }^{15} \mathrm{~N}-\left\{{ }^{1} \mathrm{H}\right\} \mathrm{NOE}$ and reference spectra were recorded with a $6 \mathrm{~s}$ saturation time for the NOE measurement and an equivalent recovery time for the reference measurement in an interleaved manner, each preceded by an additional recovery time of $1 \mathrm{~s}$. Chemical shift perturbation experiments were carried out using ${ }^{15} \mathrm{~N}$-labeled $\mathrm{PRD}_{703-800}^{\text {Strep }}$ and nonlabeled Bro1, V, and Bro1-V (one at a time). The following protein concentrations were used: $100 \mu \mathrm{M}$ $\mathrm{PRD}_{703-800}^{\text {Strep }}$ and $300 \mu \mathrm{M}$ Bro1 and V (one at a time) and $25 \mu \mathrm{M}$ $\mathrm{PRD}_{703-800}^{\mathrm{Strep}}$ and $75 \mu \mathrm{M}$ Bro1-V (the latter is due to the limited solubility of Bro1-V construct). Similar measurements were carried out using uniformly ${ }^{2} \mathrm{H} /{ }^{15} \mathrm{~N}$-labeled $100 \mu \mathrm{M}$ nonphosphorylated and phosphorylated Bro1 and nonlabeled $300 \mu \mathrm{M}$ PRD ${ }_{703-800}^{\text {Strep }}$. Perturbations were calculated as follows: $\Delta_{\mathrm{H} / \mathrm{N}}=\left\{\left(\Delta \delta_{\mathrm{HN}}\right) 2+\left(0.154 \times \Delta \delta_{\mathrm{N}}\right)^{2}\right\}^{1 / 2}$, where $\Delta \delta_{\mathrm{HN}}$ and $\Delta \delta_{\mathrm{N}}$ are the ${ }^{1} \mathrm{H}_{\mathrm{N}}$ and ${ }^{15} \mathrm{~N}$ chemical shift differences in parts per million, respectively, between free and bound states. To determine the effect of calcium on Bro1, titration experiments were carried out using ${ }^{2} \mathrm{H} /{ }^{15} \mathrm{~N}$-labeled $100 \mu \mathrm{M}$ nonphosphorylated Bro1, in the absence and presence of $5 \mathrm{mM}$ $\mathrm{CaCl}_{2}$. Intermolecular transverse ${ }^{1} \mathrm{H}_{\mathrm{N}}-\Gamma_{2}$ PRE rates were obtained by mixing $200 \mu \mathrm{M}{ }^{2} \mathrm{H} /{ }^{15} \mathrm{~N}$-labeled Bro1 with $200 \mu \mathrm{M}$ MTSL/MTS-labeled hyperphosphorylated $\mathrm{PRD}_{703-868}^{\text {Strep }}$ and its cysteine variants (note that the corresponding PRE measurements on phosphorylated Bro1 were not feasible because of the limited solubility of phosphorylated Bro1 and MTSLtagged hyperphosphorylated $\mathrm{PRD}_{703-868}^{\text {Strep }}$ mixture). Transverse ${ }^{1} \mathrm{H}_{\mathrm{N}}-\Gamma_{2}$ PRE rates were measured from the differences in the transverse ${ }^{1} \mathrm{H}_{\mathrm{N}}-R_{2}$ relaxation rates between the paramagnetic and diamagnetic samples as described previously $(26,27)$. Two time points (separated by $30 \mathrm{~ms}$ ) were used for the measurements of ${ }^{1} \mathrm{H}_{\mathrm{N}}-R_{2}$ rates, and the errors in the ${ }^{1} \mathrm{H}_{\mathrm{N}}-\Gamma_{2}$ PRE rates were calculated as described previously (26). Negative control experiments were carried out using $200 \mu \mathrm{M}{ }^{2} \mathrm{H}-/^{15} \mathrm{~N}$-labeled Bro1 and $2 \mathrm{mM}$ TEMPOL and $200 \mu \mathrm{M}$ MTSL-labeled MBPE38C. For these experiments, $200 \mu \mathrm{M}{ }^{2} \mathrm{H} /{ }^{15} \mathrm{~N}$-labeled nonphosphorylated Brol served as a diamagnetic control. NMR titration experiments between ${ }^{2} \mathrm{H}-{ }^{15} \mathrm{~N}$-labeled nonphosphorylated and phosphorylated Bro1 and neutral and negatively charged lipid vesicles were carried out using $100 \mu \mathrm{M}$ protein (protein to lipid molar ratio of 1:30). TROSY-HSQC correlation spectra were recorded on nonphosphorylated and phosphorylated Brol in the absence and presence of unilamellar lipid vesicles. The following vesicle compositions were used; the numbers in parenthesis represent the molar ratios of phospholipids: $\quad$ POPC/POPE (7:3), POPC/POPE/POPS (5.5:3:1.5), POPC/POPE/LBPA (6:3:1), POPC/POPE/LBPA (5.75:3:1.25), and POPC/POPE/LBPA (5.5:3:1.5).

\section{CR assay}

CR was dissolved in MilliQ water (MilliQ IQ 7000 purification system; Millipore-Sigma). CR stock solution $(0.2 \% \mathrm{w} / \mathrm{v})$ was filtered through a $0.22 \mu \mathrm{m}$ filter and used immediately. About $100 \mu \mathrm{M}$ solutions of nonphosphorylated and phosphorylatedPRD ${ }_{703-868}^{\text {Strep }}$ and $\mathrm{PRD}_{703-800}^{\text {Strep }}$ were incubated overnight at room temperature and mixed with CR stock solution in the morning (100:1 dilution; protein versus CR). The mixtures were incubated at room temperature for $\sim 45 \mathrm{~min}$. Absorption spectra were measured using an Agilent Cary 50 Bio UV-Vis spectrophotometer (quartz cuvette of $1 \mathrm{~cm}$ ).

\section{Fibril formation and dissolution kinetics}

Aggregation and dissolution experiments were performed at $30{ }^{\circ} \mathrm{C}$ on $\mathrm{PRD}_{703-868}^{\text {Strep }}$ samples in sealed 96-well flat bottom plates (Corning; catalog no. 3370) containing $100 \mu \mathrm{l}$ sample per well (three replicates of each sample $[n=3$ ] were placed in separate wells, and ThT fluorescence was measured through the top and bottom of the plate). Measurements were carried out with continuous linear shaking $(3.5 \mathrm{~mm}, 411.3 \mathrm{rpm})$ using a microplate reader (Infinite M Plex; Tecan). ThT (10 $\mu \mathrm{M})$ fluorescence was recorded as a function of time; excitation and emission wavelengths were 415 and $480 \mathrm{~nm}$, respectively. For aggregation experiments, ThT signals as a function of time were recorded on $100 \mu \mathrm{M}$ hyperphosphorylated $\mathrm{PRD}_{703-868}^{\text {Strep }}$ samples, in the absence and presence of $50 \mathrm{nM}$ PTP1B. The buffer conditions were as follows: $20 \mathrm{mM}$ Hepes, $\mathrm{pH} 7.5$, $1 \mathrm{mM}$ DTT, and $1 \mathrm{mM}$ EDTA. For Src-mediated fibril dissolution experiments, lyophilized $\mathrm{PRD}_{703-868}^{\text {Strep }}$ was reconstituted in a buffer containing $50 \mathrm{mM}$ Tris, $\mathrm{pH} 7.5,5 \mathrm{mM}$ $\mathrm{MgCl}_{2}, 2 \mathrm{mM}$ DTT, and $0.5 \mathrm{mM}$ EDTA $\left(\mathrm{PRD}_{703-868}^{\text {Strep }}=\right.$ $100 \mu \mathrm{M})$. ThT signals as a function of time were recorded till the samples $(n=9)$ reached a stationary phase; total incubation time of $\sim 3 \mathrm{~h}$, whereupon $1 \mu \mathrm{M}$ of recombinant Src and $1 \mathrm{mM}$ ATP were added to three samples, whereas the others were mixed with either $1 \mu \mathrm{M} \mathrm{Src}$ or $1 \mathrm{mM}$ ATP. Fibril formation of $\mathrm{PRD}_{703-868}^{\text {Strep }}$ was also assessed using ThT emission spectra recorded on Agilent Cary Eclipse Fluorescence spectrophotometer. ThT fluorescence was recorded of $3 \times 100 \mu \mathrm{l}$ of $100 \mu \mathrm{M} \mathrm{PRD}_{703-868}^{\text {Strep }}$ samples at $30{ }^{\circ} \mathrm{C}$ after $\sim 2 \mathrm{~h}$ incubation (20 mM Hepes, pH 7.5, 1 mM DTT, and 1 mM EDTA; micro quartz cuvettes). Similar measurements were recorded on hyperphosphorylated $\mathrm{PRD}_{703-868}^{\text {Strep }}$ and the soluble N-terminal fragment, $\mathrm{PRD}_{703-800}^{\mathrm{Strep}}$, which served as negative controls.

\section{TEM}

PRD ${ }_{703-868}^{\text {Strep }}$ samples, $\sim 1.2 \mathrm{mg} / \mathrm{ml}$, were incubated at $30^{\circ} \mathrm{C}$ in $20 \mathrm{mM}$ sodium phosphate, $\mathrm{pH} 7.0,50 \mathrm{mM} \mathrm{NaCl}, 1 \mathrm{mM}$ TCEP, and $1 \mathrm{mM}$ EDTA. Aggregated samples were subjected to 


\section{Structure-function studies of tyrosine phosphorylation of ALIX}

sonication ( $10 \mathrm{~min}$; Elmasonic P ultrasonic bath) and diluted to $\sim 0.2 \mathrm{mg} / \mathrm{ml}$ immediately before application to the TEM grids (400-mesh formvar and carbon-coated copper; Electron Microscopy Sciences; catalog no. FCF400-Cu). About $1 \mathrm{~min}$ after deposition, the sample solution was wicked with filter paper, followed by a quick wash with $3 \mu \mathrm{l}$ of water and the addition of three to five drops of $2 \% \mathrm{w} / \mathrm{v}$ aqueous uranyl acetate solution. The uranyl acetate was wicked immediately with a filter paper, and the grids were air dried at room temperature. TEM images were acquired using a JEOL JEM1400Plus transmission electron microscope (JEOL) and recorded on a Gatan OneView digital camera (Gatan).

\section{CD spectroscopy}

CD measurements $(178-280 \mathrm{~nm}, 1 \mathrm{~nm}$ data pitch, continuous scanning with $1 \mathrm{~nm}$ bandwidth, $60 \mathrm{~nm} / \mathrm{min}$, and ten accumulations) with $0.1 \mathrm{mg} / \mathrm{ml}$ nonphosphorylated and phosphorylated $\mathrm{PRD}_{703-868}^{\text {Strep }}$ were carried out in $2 \mathrm{~mm}$ quartz cuvettes (Starna Cells, Inc) using an Aviv model 215 spectrometer. The molar ellipticity was calculated using the equation: $[\theta]_{\text {molar }}=100 * \mathrm{MW} * \theta / \mathrm{cl}$, where $\mathrm{MW}=$ molecular weight, $\theta=\mathrm{CD}$ signal (mdeg), $\mathrm{c}=$ concentration $(\mathrm{mg} / \mathrm{ml})$, and $\mathrm{l}=$ path length $(\mathrm{cm})$. The buffer and experimental conditions were as follows: $10 \mathrm{mM}$ sodium phosphate, $\mathrm{pH}$ 7.0, $1 \mathrm{mM}$ TCEP, $1 \mathrm{mM}$ EDTA, and $25^{\circ} \mathrm{C}$.

\section{Data availability}

Plasmids of full-length ALIX-PRD, PRD Strep $_{7068}$, and its cysteine variants, namely $\mathrm{PRD}_{703-868}^{\text {Strep }}-\mathrm{S} 712 \mathrm{C}, \mathrm{C} 813 \mathrm{~S}$, $\mathrm{PRD}_{703-868}^{\text {Strep }}-\mathrm{A} 756 \mathrm{C}, \mathrm{C} 813 \mathrm{~S}$, and PRD $\mathrm{PR}_{703-868}^{\text {Strep }}-\mathrm{S} 863 \mathrm{C}, \mathrm{C} 813 \mathrm{~S}$, have been deposited in the Addgene repository, https://www. addgene.org/ (accession nos. 164444, 164445, 164446, and 164447, respectively). The chemical shift assignments of Bro1 have been deposited in the Biological Magnetic Resonance Bank, https://bmrb.io/ (accession no. 50707). LC-MS/MS data were deposited to the MassIVE data repository, https://massive.ucsd. edu/ (accession no. MSV000088057).

Supporting information-This article contains supporting information $(16,22,23,26,27,60-64)$.

Acknowledgments-We thank Z. Jiang, R. Ghirlando, P. A. Jennings, and I. Budin for useful discussions; X. Huang (University of California San Diego [UCSD] Biomolecular NMR facility), L. Gross, and Y. Su (UCSD Molecular Mass Spectrometry Facility) and G. Castillon and T. Meerloo (UCSD Cellular and Molecular Medicine Electron Microscopy Core Facility) for technical support. We thank A. van der Vliet (University of Vermont), D. S. Waugh (National Cancer Institute, NIH), and G. Marius Clore (National Institute of Diabetes and Digestive and Kidney Diseases, NIH) for Src kinase, TEV protease, and apo-MBP-E38C constructs, respectively. We acknowledge the use of facilities and instrumentation supported by the National Science Foundation through the UCSD Materials Research Science and Engineering Center, DMR-2011924, and by the NIH for the UCSD Cellular and Molecular Medicine EM Core Facility; S10OD023527, and the UCSD Molecular Mass Spectrometry Facility; 1S10RR25636-1A1.
Author contributions-R. D. E, B. R., and L. D. methodology; R. D. E, B. R., and L. D. investigation; R. D. E, B. R., and L. D. formal analysis; R. D. E and L. D. writing-original draft.

Funding and additional information-This work was funded in part by a 2018 Development Award (to L. D.) from the San Diego Center for AIDS Research (an NIH-funded program; P30 AI036214) and by Collaborative Development Awards (to L. D.) from the Cellular Host Elements in Egress, Trafficking, and Assembly of HIV (CHEETAH Center, University of Utah; an NIH-funded program; P50 AI150464) and HIV Interactions in Viral Evolution (HIVE Center, the Scripps research institute; an NIH-funded program; U54 AI150472), and a National Science Foundation Graduate Research Fellowship under grant no. DGE-1650112 (to R. D. E.). The content is solely the responsibility of the authors and does not necessarily represent the official views of the National Institutes of Health.

Conflict of interest-The authors declare that they have no conflicts of interest with the contents of this article.

Abbreviations-The abbreviations used are: ALG-2, apoptosis linked gene-2; ALIX, apoptosis-linked gene-2 interacting protein X; $\mathrm{CD}$, circular dichroism; CEP55, centrosomal protein of $55 \mathrm{kDa}$; CHMP, charged multivesicular body protein; CIL, Cambridge Isotopes Laboratories; CR, congo red; DLS, dynamic light scattering; $\mathrm{D}_{2} \mathrm{O}$, deuterium oxide; ESI, electrospray ionization; GB1, B1 domain of protein G; HSQC, heteronuclear single quantum coherence; LBPA, 2,2' lysobisphosphatidic acid; LC, liquid chromatography; MBP, maltose-binding protein; MS, mass spectrometry; MTS, (1-acetoxy-2,2,5,5-tetramethyl- $\delta$-3-pyrroline-3-methyl) methanethiosulfonate; MTSL, (1-oxyl-2,2,5,5-tetramethyl- $\Delta 3$ pyrroline-3-methyl) methanethiosulfonate; NIH, National Institutes of Health; PK, proteinase K; POPC, 1-palmitoyl-2-oleoylglycero-3-phosphocholine; POPE, 1-palmitoyl-2-oleoyl-sn-glycero3-phosphoethanolamine; POPS, 1-palmitoyl-2-oleoyl-sn-glycero-3phospho-L-serine; PRD, proline-rich domain; PRE, paramagnetic relaxation enhancement; PTM, post-translational modification; PTP1B, protein-tyrosine phosphatase 1B; TEM, transmission electron microscopy; TEMPOL, 4-hydroxy-2,2,6,6-tetramethylpiperidine 1-oxyl; TEV, tobacco etch virus; ThT, thioflavin T; TOF, time-offlight; TROSY, transverse relaxation optimized spectroscopy; UCSD, University of California San Diego; UEV, ubiquitin E2 variant.

\section{References}

1. Odorizzi, G. (2006) The multiple personalities of Alix. J. Cell Sci. 119, 3025-3032

2. Sadoul, R. (2006) Do Alix and ALG-2 really control endosomes for better or for worse? Biol. Cell 98, 69-77

3. Hurley, J. H., and Odorizzi, G. (2012) Get on the exosome bus with ALIX. Nat. Cell Biol. 14, 654-655

4. Bissig, C., and Gruenberg, J. (2014) ALIX and the multivesicular endosome: ALIX in Wonderland. Trends Cell Biol. 24, 19-25

5. Strack, B., Calistri, A., Craig, S., Popova, E., and Gottlinger, H. G. (2003) AIP1/ALIX is a binding partner for HIV-1 p6 and EIAV p9 functioning in virus budding. Cell 114, 689-699

6. von Schwedler, U. K., Stuchell, M., Muller, B., Ward, D. M., Chung, H. Y., Morita, E., Wang, H. E., Davis, T., He, G. P., Cimbora, D. M., Scott, A., Krausslich, H. G., Kaplan, J., Morham, S. G., and Sundquist, W. I. (2003) The protein network of HIV budding. Cell 114, 701-713

7. Han, Z., Madara, J. J., Liu, Y., Liu, W., Ruthel, G., Freedman, B. D., and Harty, R. N. (2015) ALIX rescues budding of a double PTAP/PPEY L-domain deletion mutant of ebola VP40: A role for ALIX in ebola virus egress. J. Infect. Dis. 212 Suppl 2, S138-S145 


\section{Structure-function studies of tyrosine phosphorylation of ALIX}

8. Schmidt, M. H. H., Dikic, I., and Bogler, O. (2005) Src phosphorylation of Alix/AIP1 modulates its interaction with binding partners and antagonizes its activities. J. Biol. Chem. 280, 3414-3425

9. Hikita, T., Kuwahara, A., Watanabe, R., Miyata, M., and Oneyama, C. (2019) Src in endosomal membranes promotes exosome secretion and tumor progression. Sci. Rep. 9, 3265

10. Matsuo, H., Chevallier, J., Mayran, N., Le Blanc, I., Ferguson, C., Fauré, J., Blanc, N. S., Matile, S., Dubochet, J., Sadoul, R., Parton, R. G., Vilbois, F., and Gruenberg, J. (2004) Role of LBPA and Alix in multivesicular liposome formation and endosome organization. Science 303, 531-534.

11. Bissig, C., Lenoir, M., Velluz, M. C., Kufareva, I., Abagyan, R., Overduin, M., and Gruenberg, J. (2013) Viral infection controlled by a calciumdependent lipid-binding module in ALIX. Dev. Cell 25, 364-373

12. Kobayashi, T., Stang, E., Fang, K. S., de Moerloose, P., Parton, R. G., and Gruenberg, J. (1998) A lipid associated with the antiphospholipid syndrome regulates endosome structure and function. Nature 392, 193-197

13. Zhou, X., Si, J., Corvera, J., Gallick, G. E., and Kuang, J. (2010) Decoding the intrinsic mechanism that prohibits ALIX interaction with ESCRT and viral proteins. Biochem. J. 432, 525-534

14. Zhai, Q., Landesman, M. B., Chung, H.-Y., Dierkers, A., Jeffries, C. M., Trewhella, J., Hill, C. P., and Sundquist, W. I. (2011) Activation of the retroviral budding factor ALIX. J. Virol. 85, 9222-9226

15. Ren, X., and Hurley, J. H. (2011) Proline-rich regions and motifs in trafficking: From ESCRT interaction to viral exploitation. Traffic 12, $1282-1290$

16. Elias, R. D., Ma, W., Ghirlando, R., Schwieters, C. D., Reddy, V. S., and Deshmukh, L. (2020) Proline-rich domain of human ALIX contains multiple TSG101-UEV interaction sites and forms phosphorylationmediated reversible amyloids. Proc. Natl. Acad. Sci. U. S. A. 117, 24274-24284

17. Schmidt, T. G., and Skerra, A. (2007) The Strep-tag system for one-step purification and high-affinity detection or capturing of proteins. Nat. Protoc. 2, 1528-1535

18. Huth, J. R., Bewley, C. A., Jackson, B. M., Hinnebusch, A. G., Clore, G. M., and Gronenborn, A. M. (1997) Design of an expression system for detecting folded protein domains and mapping macromolecular interactions by NMR. Protein Sci. 6, 2359-2364.

19. Tsiatsiani, L., and Heck, A. J. (2015) Proteomics beyond trypsin. FEBS J. 282, 2612-2626

20. Baudier, J., and Cole, R. D. (1987) Phosphorylation of tau proteins to a state like that in Alzheimer's brain is catalyzed by a calcium/calmodulindependent kinase and modulated by phospholipids. J. Biol. Chem. 262, 17577-17583

21. Stuible, M., Abella, J. V., Feldhammer, M., Nossov, M., Sangwan, V., Blagoev, B., Park, M., and Tremblay, M. L. (2010) PTP1B targets the endosomal sorting machinery: Dephosphorylation of regulatory sites on the endosomal sorting complex required for transport component STAM2. J. Biol. Chem. 285, 23899-23907

22. Shen, Y., and Bax, A. (2013) Protein backbone and sidechain torsion angles predicted from NMR chemical shifts using artificial neural networks. J. Biomol. NMR 56, 227-241

23. Sadler, J. B. A., Wenzel, D. M., Williams, L. K., Guindo-Martínez, M., Alam, S. L., Mercader, J. M., Torrents, D., Ullman, K. S., Sundquist, W. I., and Martin-Serrano, J. (2018) A cancer-associated polymorphism in ESCRT-III disrupts the abscission checkpoint and promotes genome instability. Proc. Natl. Acad. Sci. U. S. A. 115, E8900-E8908

24. Kay, B. K., Williamson, M. P., and Sudol, M. (2000) The importance of being proline: The interaction of proline-rich motifs in signaling proteins with their cognate domains. FASEB J. 14, 231-241

25. Zarrinpar, A., Bhattacharyya, R. P., and Lim, W. A. (2003) The structure and function of proline recognition domains. Sci. STKE 2003, RE8

26. Clore, G. M., and Iwahara, J. (2009) Theory, practice, and applications of paramagnetic relaxation enhancement for the characterization of transient low-population states of biological macromolecules and their complexes. Chem. Rev. 109, 4108-4139

27. Deshmukh, L., Louis, J. M., Ghirlando, R., and Clore, G. M. (2016) Transient HIV-1 Gag-protease interactions revealed by paramagnetic
NMR suggest origins of compensatory drug resistance mutations. Proc. Natl. Acad. Sci. U. S. A. 113, 12456-12461

28. Baker, N. A., Sept, D., Joseph, S., Holst, M. J., and McCammon, J. A. (2001) Electrostatics of nanosystems: Application to microtubules and the ribosome. Proc. Natl. Acad. Sci. U. S. A. 98, 10037-10041

29. Rideau, E., Dimova, R., Schwille, P., Wurm, F. R., and Landfester, K. (2018) Liposomes and polymersomes: A comparative review towards cell mimicking. Chem. Soc. Rev. 47, 8572-8610

30. Carlton, J. G., Agromayor, M., and Martin-Serrano, J. (2008) Differential requirements for Alix and ESCRT-III in cytokinesis and HIV-1 release. Proc. Natl. Acad. Sci. U. S. A. 105, 10541-10546

31. Trioulier, Y., Torch, S., Blot, B., Cristina, N., Chatellard-Causse, C., Verna, J. M., and Sadoul, R. (2004) Alix, a protein regulating endosomal trafficking, is involved in neuronal death. J. Biol. Chem. 279, 204.6-2052

32. Kamranvar, S. A., Gupta, D. K., Huang, Y., Gupta, R. K., and Johansson, S. (2016) Integrin signaling via FAK-Src controls cytokinetic abscission by decelerating PLK1 degradation and subsequent recruitment of CEP55 at the midbody. Oncotarget 7, 30820-30830

33. Shibata, H., Yamada, K., Mizuno, T., Yorikawa, C., Takahashi, H., Satoh, H., Kitaura, Y., and Maki, M. (2004) The penta-EF-hand protein ALG-2 interacts with a region containing PxY repeats in Alix/AIP1, which is required for the subcellular punctate distribution of the amino-terminal truncation form of Alix/AIP1. J. Biochem. 135, 117-128

34. Schöneberg, J., Lee, I.-H., Iwasa, J. H., and Hurley, J. H. (2017) Reversetopology membrane scission by the ESCRT proteins. Nat. Rev. Mol. Cell Biol. 18, 5-17

35. Vietri, M., Radulovic, M., and Stenmark, H. (2020) The many functions of ESCRTs. Nat. Rev. Mol. Cell Biol. 21, 25-42

36. Christ, L., Wenzel, E. M., Liestøl, K., Raiborg, C., Campsteijn, C., and Stenmark, H. (2016) ALIX and ESCRT-I/II function as parallel ESCRT-III recruiters in cytokinetic abscission. J. Cell Biol. 212, 499-513

37. Larios, J., Mercier, V., Roux, A., and Gruenberg, J. (2020) ALIX- and ESCRT-III-dependent sorting of tetraspanins to exosomes. J. Cell Biol. 219

38. Kim, J., Sitaraman, S., Hierro, A., Beach, B. M., Odorizzi, G., and Hurley, J. H. (2005) Structural basis for endosomal targeting by the Bro1 domain. Dev. Cell 8, 937-947

39. Chiti, F., and Dobson, C. M. (2017) Protein misfolding, amyloid formation, and human disease: A summary of progress over the last decade. Annu. Rev. Biochem. 86, 27-68

40. Hammer, N. D., Wang, X., McGuffie, B. A., and Chapman, M. R. (2008) Amyloids: Friend or foe? J. Alzheimers Dis. 13, 407-4.19

41. Jackson, M. P., and Hewitt, E. W. (2017) Why are functional amyloids non-toxic in humans? Biomolecules 7, 71

42. Wang, M., Audas, T. E., and Lee, S. (2017) Disentangling a bad reputation: Changing perceptions of amyloids. Trends Cell Biol. 27, 465-4.67

43. Otzen, D., and Riek, R. (2019) Functional amyloids. Cold Spring Harb. Perspect. Biol. 11, a033860

44. Khoury, G. A., Baliban, R. C., and Floudas, C. A. (2011) Proteome-wide post-translational modification statistics: Frequency analysis and curation of the swiss-prot database. Sci. Rep. 1, 90

45. Chiti, F., and Dobson, C. M. (2006) Protein misfolding, functional amyloid, and human disease. Annu. Rev. Biochem. 75, 333-366

46. Schnabel, J. (2010) Protein folding: The dark side of proteins. Nature 464, 828-829

47. McCullough, J., Fisher, R. D., Whitby, F. G., Sundquist, W. I., and Hill, C. P. (2008) ALIX-CHMP4 interactions in the human ESCRT pathway. Proc. Natl. Acad. Sci. U. S. A. 105, 7687-7691

48. Lee, S., Joshi, A., Nagashima, K., Freed, E. O., and Hurley, J. H. (2007) Structural basis for viral late-domain binding to Alix. Nat. Struct. Mol. Biol. 14, 194-199

49. Carlson, L. A., and Hurley, J. H. (2012) In vitro reconstitution of the ordered assembly of the endosomal sorting complex required for transport at membrane-bound HIV-1 Gag clusters. Proc. Natl. Acad. Sci. U. S. A. 109, 16928-16933

50. Choy, M. S., Li, Y., Machado, L., Kunze, M. B. A., Connors, C. R., Wei, X., Lindorff-Larsen, K., Page, R., and Peti, W. (2017) Conformational rigidity 
and protein dynamics at distinct timescales rgulate PTP1B activity and allostery. Mol. Cell 65, 644-658.e645

51. Ramaraju, B., Nelson, S. L., Zheng, W., Ghirlando, R., and Deshmukh, L. (2021) Quantitative NMR study of insulin-degrading enzyme using amyloid- $\beta$ and HIV-1 p6 elucidates its chaperone activity. Biochemistry 60, $2519-2523$

52. Zhang, Z., and Marshall, A. G. (1998) A universal algorithm for fast and automated charge state deconvolution of electrospray mass-to-charge ratio spectra. J. Am. Soc. Mass Spectrom. 9, 225-233

53. Rost, H. L., Sachsenberg, T., Aiche, S., Bielow, C., Weisser, H., Aicheler, F., Andreotti, S., Ehrlich, H. C., Gutenbrunner, P., Kenar, E., Liang, X., Nahnsen, S., Nilse, L., Pfeuffer, J., Rosenberger, G., et al. (2016) OpenMS: A flexible open-source software platform for mass spectrometry data analysis. Nat. Methods 13, 74.1-74.8

54. Kim, S., and Pevzner, P. A. (2014) MS-GF+ makes progress towards a universal database search tool for proteomics. Nat. Commun. 5, 5277

55. Jiang, Z., de Messieres, M., and Lee, J. C. (2013) Membrane remodeling by $\alpha$-synuclein and effects on amyloid formation. J. Am. Chem. Soc. 135, 15970-15973

56. Delaglio, F., Grzesiek, S., Vuister, G. W., Zhu, G., Pfeifer, J., and Bax, A. (1995) NMRPipe: A multidimensional spectral processing system based on UNIX pipes. J. Biomol. NMR 6, 277-293

57. Vranken, W. F., Boucher, W., Stevens, T. J., Fogh, R. H., Pajon, A., Llinas, M., Ulrich, E. L., Markley, J. L., Ionides, J., and Laue, E. D. (2005) The CCPN data model for NMR spectroscopy: Development of a software pipeline. Proteins 59, 687-696
58. Clore, G. M., and Gronenborn, A. M. (1998) Determining the structures of large proteins and protein complexes by NMR. Trends Biotechnol. 16, 22-34.

59. Lakomek, N. A., Ying, J., and Bax, A. (2012) Measurement of ${ }^{15} \mathrm{~N}$ relaxation rates in perdeuterated proteins by TROSY-based methods. J. Biomol. NMR 53, 209-221

60. Roepstorff, P., and Fohlman, J. (1984) Proposal for a common nomenclature for sequence ions in mass spectra of peptides. Biomed. Mass Spectrom. 11, 601

61. Brademan, D. R., Riley, N. M., Kwiecien, N. W., and Coon, J. J. (2019) Interactive peptide spectral annotator: A versatile web-based tool for proteomic applications. Mol. Cell. Proteomics 18, S193-S201

62. Zhang, Z. Y., Thieme-Sefler, A. M., Maclean, D., McNamara, D. J., Dobrusin, E. M., Sawyer, T. K., and Dixon, J. E. (1993) Substrate specificity of the protein tyrosine phosphatases. Proc. Natl. Acad. Sci. U. S. A. $90,4446-4450$

63. Duan, X., Hall, J. A., Nikaido, H., and Quiocho, F. A. (2001) Crystal structures of the maltodextrin/maltose-binding protein complexed with reduced oligosaccharides: Flexibility of tertiary structure and ligand binding. J. Mol. Biol. 306, 1115-1126

64. Ferreira, C. M. H., Pinto, I. S. S., Soares, E. V., and Soares, H. M. V. M. (2015) (Un)suitability of the use of pH buffers in biological, biochemical and environmental studies and their interaction with metal ions - a review. RSC Adv. 5, 30989-31003

65. Schwieters, C. D., Kuszewski, J. J., Tjandra, N., and Clore, G. M. (2003) The Xplor-NIH NMR molecular structure determination package. J. Magn. Reson. 160, 65-73 